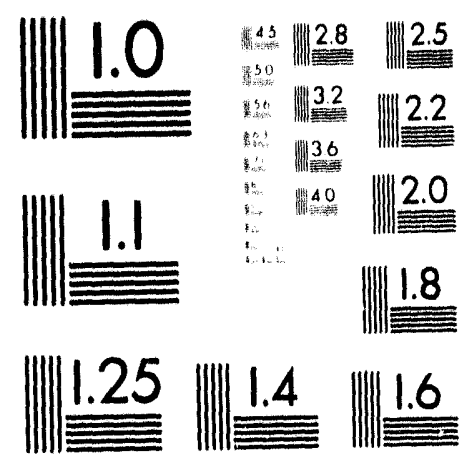



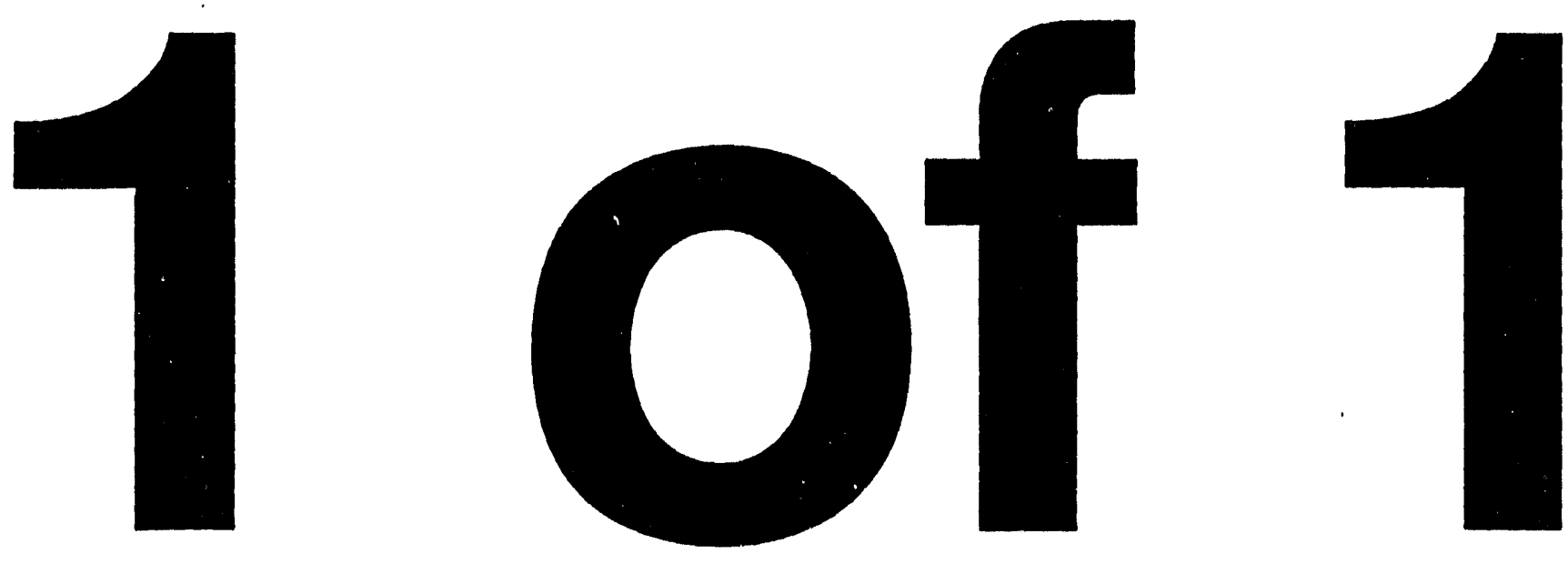


\title{
Potential Application of the Motorola MSR-20 Radar to DOE Site Security*
}

\author{
Duane Arlowe \\ Pete Rebeil \\ Richard Vigil \\ Nuclear Security Systems Center \\ Sandia National Laboratories 9500 \\ Albuquerque, New Mexico 87185
}

\begin{abstract}
This paper describes the results of testing the MSR-20 radar and provides guidance on how this radar may be used to provide early detection and warning of approaching intruders beyond DOE facility site boundaries.

*This work was supported by DOE Headquarters, Office of Safeguards and Security, under contract \#SNL89034
\end{abstract}




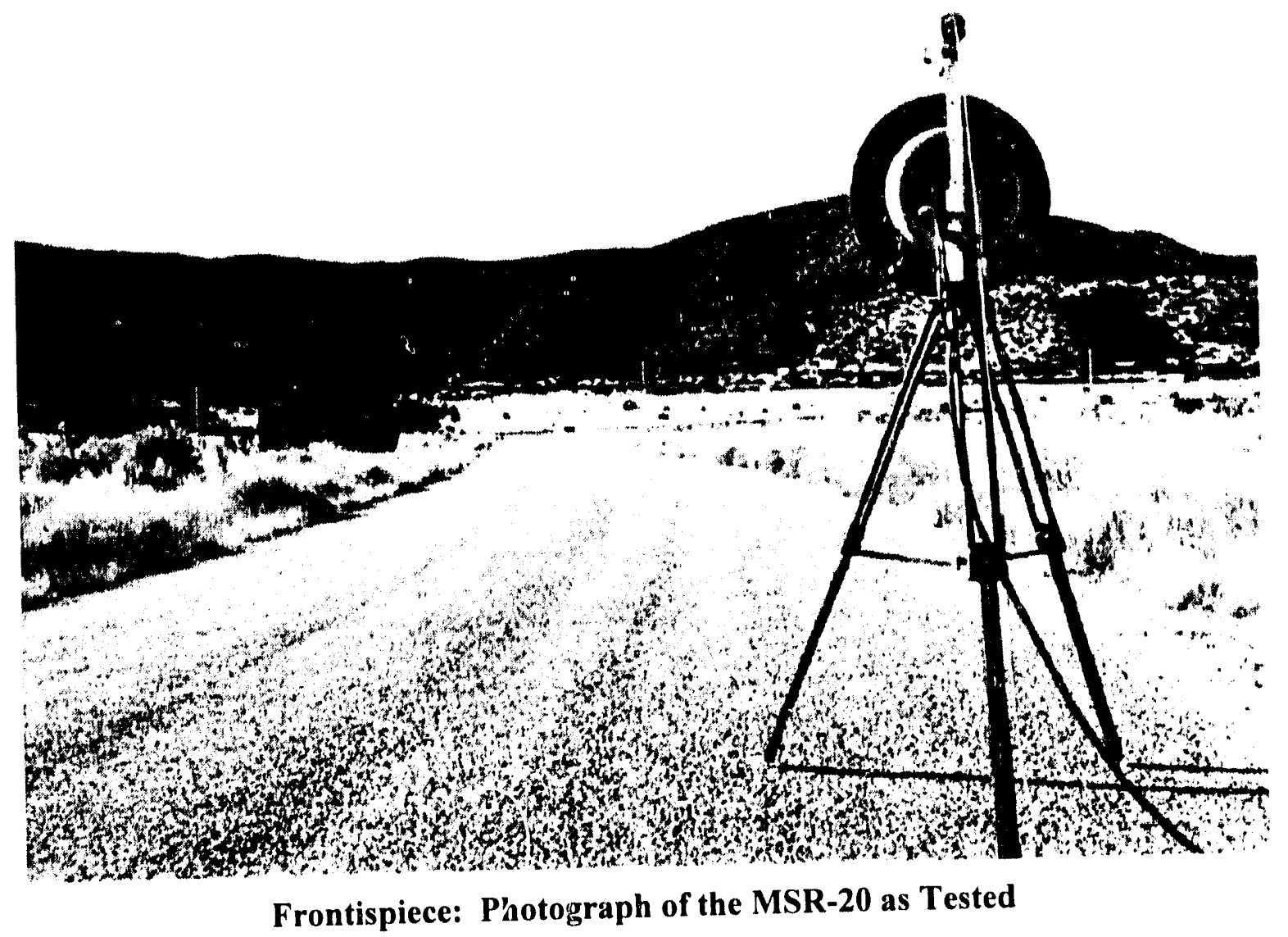




\section{Executive Summary}

The addition of an early warning detection system to current DOE site security has been suggested by security managers as a needed and valuable upgrade. This concept would increase protection in depth, and would allow additional guard response time to intruders about to breach the outer security fence. Such a system may also detect terrorists with stand-off weapons, thus providing the only warning of this kind of attack.

With these applications in mind, Sandia performed a limited test and evaluation of the Motorola MSR-20. This portable pulsed Doppler radar was developed for the military to warn of approaching enemy troops, especially at night. Although we found that it would detect even single personnel at long range as advertised, it is subject to false alarms due to animals and blowing vegetation. It is designed for operation by dedicated and trained personnel.

For application to DOE sites, a rapid and positive alarm assessment capability such as IR and CCTV cameras must be added. This radar would seem to have its best application to isolated sites in the western US where there is little ground cover and flat terrain. Since it can cover thousands of acres, there may be a high nuisance alarm rate from animals. Also, the false alarms from blowing vegetation may cause the operator to raise the detection threshold to the point where detection of humans is no longer probable.

Finally, this kind of radar could not be expected to provide complete coverage alone. Certain areas, such as approaches near access roads with uncontrolled traffic, and hidden areas, such as depressions and arroyos, will have additional, localized sensors. The terrain and ground cover may need to be modified, the animal population controlled, and access routes revised.

Any site that is considering the use of the MSR-20 or any similar radar for early warning should conduct a specific site systems analysis that covers all of the issues identified in this study. 


\section{Contents}

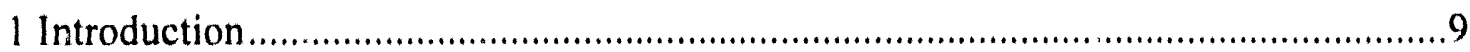

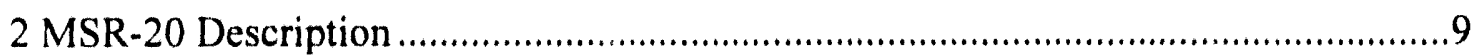

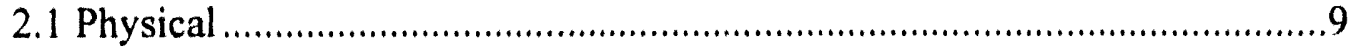

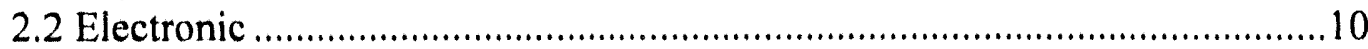

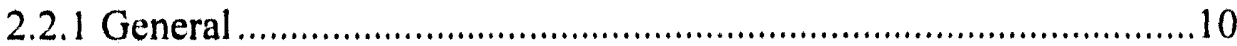

2.2.2 Target Range ........................................................................

2.2.3 Target Velocity …………………............................................11

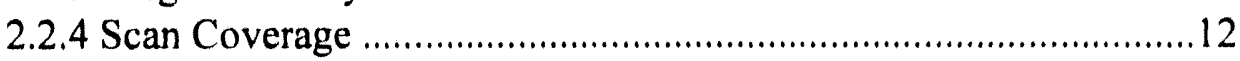

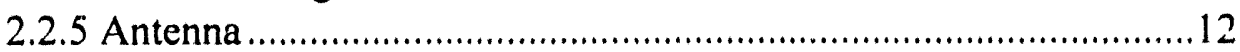

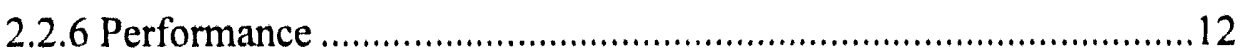

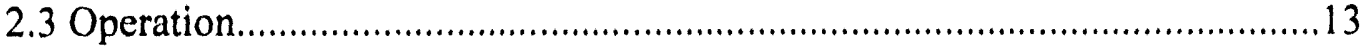

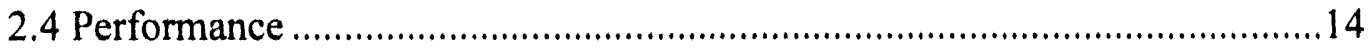

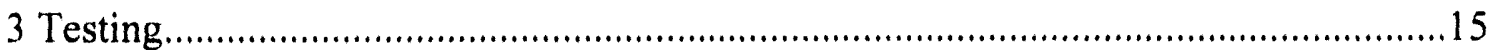

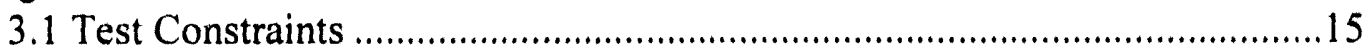

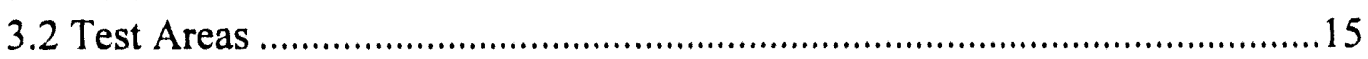

3.3 Test Targets.........................................................................................15

3.4 Summary of Test Results ....................................................................15

3.4.1 Test Series I (3900 Meters) ..........................................................15

3.4.2 Test Series II (4600 Meters).........................................................18

3.4.3 Test Series III (2600 Meters) ....................................................18

3.4.4 Test Series IV (550 Meters in Light Brush)...................................22

3.4.5 Assessment Test (Series V) ……………………............................24

3.4.5.1 Human Intruder Assessment ............................................24

3.4.5.2 Fast Truck Assessment ....................................................24

3.4.6 Performance Vs. Antenna Height Above Ground (Series VI) ........25

3.4.7 Performance Vs. Antenna Beamwidth (Series VII) .........................26

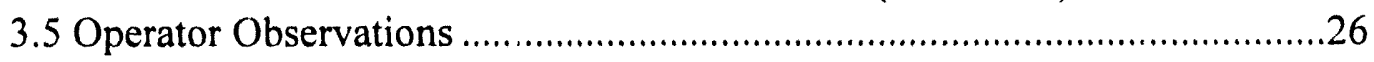

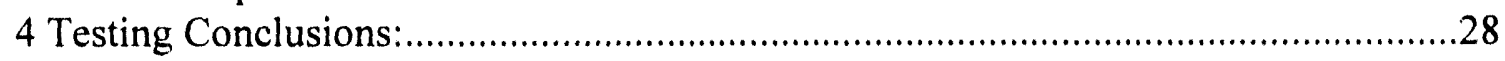

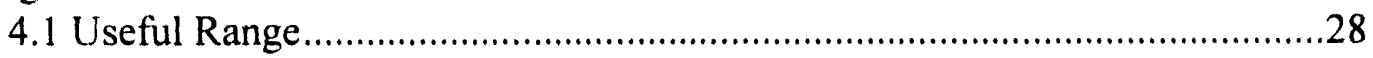

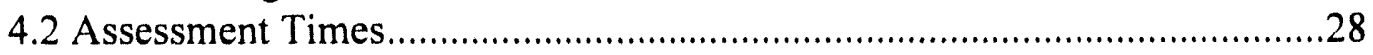

4.3 Designating Access Areas.........................................................................29

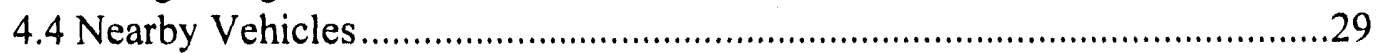

4.5 Near Range Boundary ………………………………..........................29

4.6 Distant Targets .........................................................................................

4.7 Sector Edges...........................................................................................

4.8 Antenna Beam Geometry ........................................................................31

4.8.1 Available Beam Widths ............................................................31

4.8.2 Pointing Assessment Cameras .........................................................31

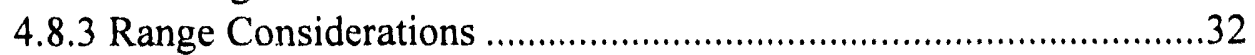

4.9 Antenna Azimuth Positioning …….............................................................32

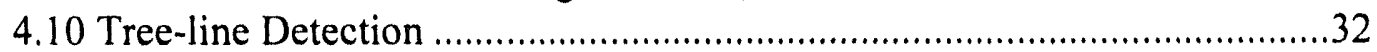

4.11 Additional General Notes and Recommendations .....................................32

5. Expected Battlefield Performance ........................................................................... 


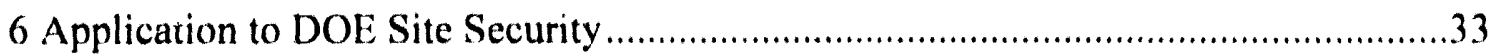

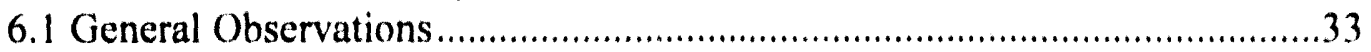

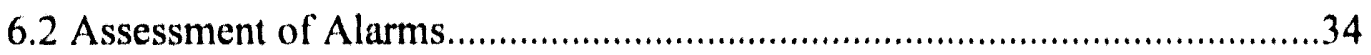

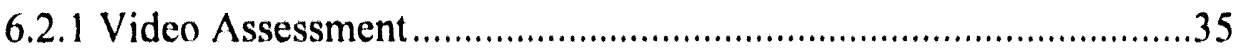

6.2.2 Thermal Image Assessment at Night ........................................35

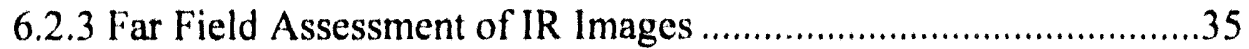

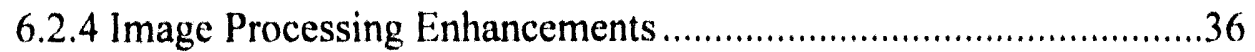

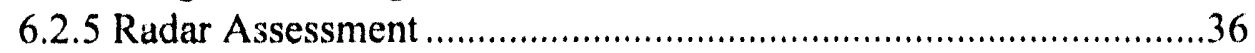

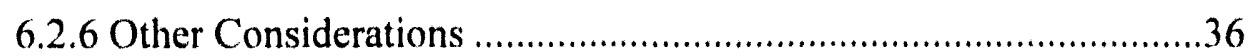

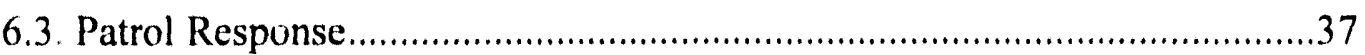

6.4 Guidelines for Selecting Sites Suitable for Radar Use ...............................38

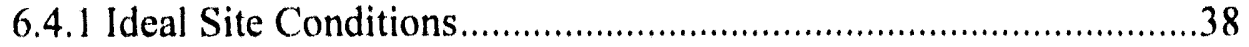

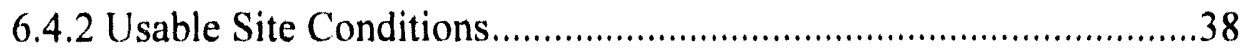

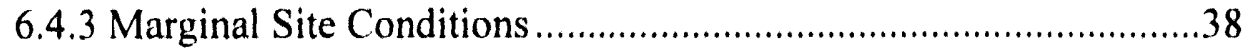

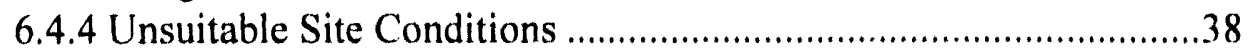

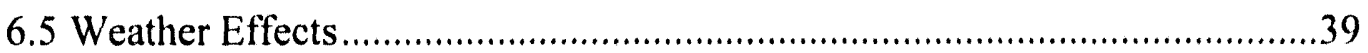

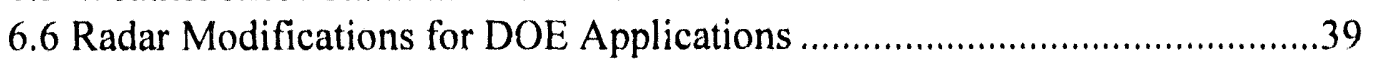

6.6.1 Proposed Operation with Unmodified Radar................................ 39

6.6.2 Proposed Operation with Modified Radar. ....................................40

6.6.3 Fully Integrated Radar System........................................................41

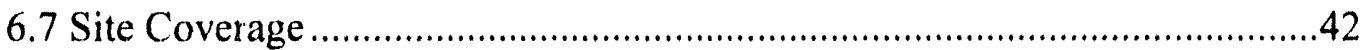

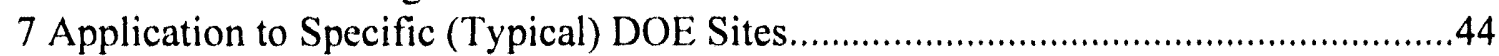

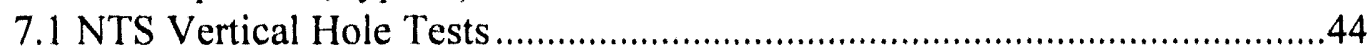

7.2 NTS Tunnel Shots............................................................................45

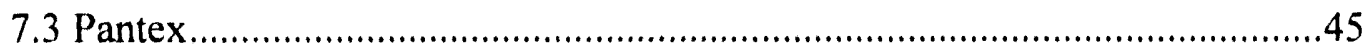

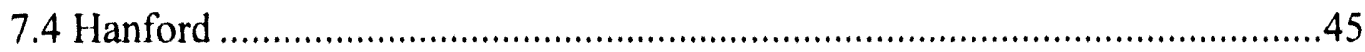

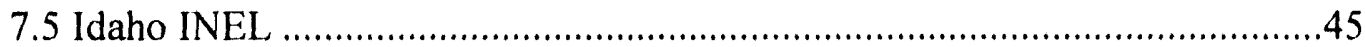

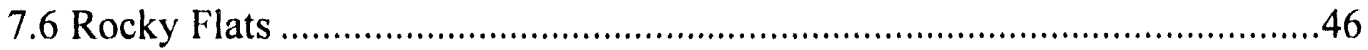

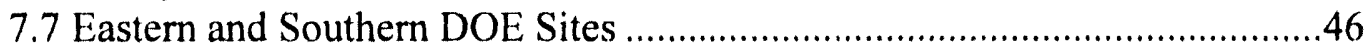

8 Ability to Detect Terrorists with Stand-off Weapons .................................................46

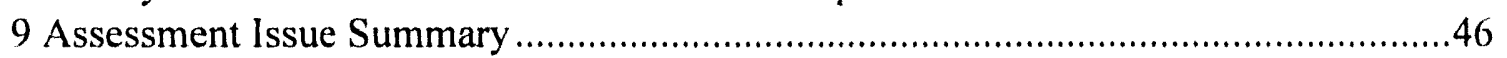

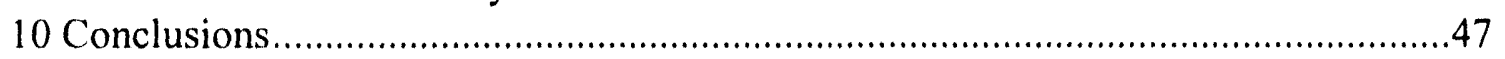




\section{Figures}

1. Block Diagram of MSR-20 Radar ....................................................... 10

2. Velocity-Folding Diagram ......................................................... 12

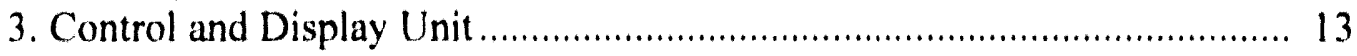

4. Map of the ESL Test Range ....................................................... 16

5. $19 \mathrm{p}$ of the RVR Test Range

6. 1 est Towers Used in Testing the MSR-20........................................ 19

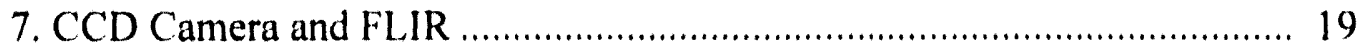

8. MSR-20 Looking South Over the RVR .............................................. 20

9. Test Tower and Radar ........................................................................ 20

10. Plot of True and False Alarm Rates Vs. Threshold ................................ 21

11. Human Target Hidden by Cactus..................................................... 22

12. Radar Set Up to Detect Personnel in Tree Line ....................................... 23

13. The Radar Test Range at Coyote Canyon.............................................. 23

14. Plot of Pd Vs Antenna Height at the RVR ............................................ 25

15. Distortion of the Intruder's Path ............................................................ 27

16. Radar Pattern Placements Near Access Road ......................................... 30

17. Search Areas Needed at Different Radar Ranges ................................... 37

18. Block Diagram of a Centralized Alarm Processor and True Map Display. 41

19. Radar Location and Coverage for Typical Sites ..................................... 43

20. Single Radar on Tall Tower..................................................................... 43

21. Radar Screen to Reduce False Alarms from Perimeter Traffic.................. 44

\section{Tables}

1. Target Detection Modes................................................................. 10

2. MSR-20 Detection Specification. .......................................................... 13

3. Comparison of DOE and DoD Needs .................................................... 34

4. Expected Range Required for Assessment ............................................. 36 


\section{Potential Application of the Motorola MSR-20 Radar to DOE Site Security}

\section{Introduction}

The purpose of this evaluation is to determine the advantages and disadvantages of using the Motorola MSR-20 (and perhaps other similar grcund search radars) at applicable DOE sites to achieve the following:

- Early warning of approaching land-based intruders before they arrive at an alarmed perimeter; and early detection of land-based intruders where there is no alarmed perimeter (e.g., NTS ground zeros). Early warning in this case implies at least 1-2 minutes of warning time, or at least a 1-2 km detection range (whichever is greater).

- Detection of personnel who may be searching for a location from which to use a standoff weapon against a facility.

The testing of the MSR-20 radar by Sandia for this project was necessarily limited. We could have confirmed that this radar met its design parameters by testing it in an anechoic radar chamber using assumed equivalent radar cross section targets that had been reduced in size to simulate signal reduction due to range. This would tell us if the radar meets design performance specifications, but would tell us little about its practical use.

Field testing reveals a lot about the type of real world problems that appear when using a radar in a typical site environment. Such testing, to be truly complete and repeatable, must be done under very controlled conditions. It is hard to control, measure, or repeat the radar signals that one would get from the target and the background, especially with the wind blowing. If the target, background, and wind conditions were the same between two tests, but other variables such as the contour of the terrain and the radar location relative to the ground and nearby buildings were different, the probability of detection may also be different. Detection performance can also vary due to multi-path reflections or other little understood effects.

Consequently, we resorted to anecdotal testing, where we did specific testing under what appeared to us to be typical situations and applications. The scope of this project precluded identifying specific sites for the testing and application of this radar.

\section{MSR-20 Description}

\subsection{Physical}

This Motorola Surveillance Radar is a pulsed Doppler system operating in the X-band. It is a man-portable system composed of three major components and three cables as shown in the block diagram (Figure 1).

- the antenna-receiver-transmitter (ART), usually mounted on a tripod,

- a signal processor unit (SPU),

- a control display unit (CDU) including a liquid crystal display and a water resistant keypad. 


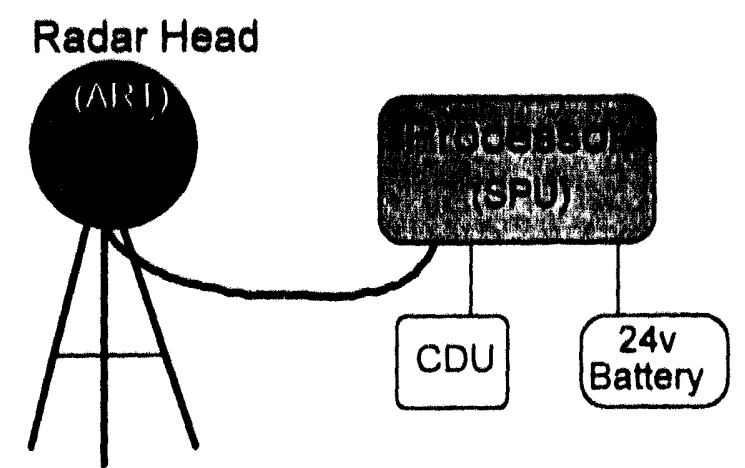

Figure 1. Block Diagram of MSR-20 Radar

\subsection{Electronic}

\subsubsection{General}

The MSR-20 radar operates at a $9.46 \mathrm{MHz}$ carrier frequency and a pulse repetition frequency (PRF) of about 1800 per second. This frequency range is very popular for ground surveillance radars because higher frequencies result in systems that are more expensive and whose performance is degraded by rain and fog. Lower frequencies yield unacceptably large and slowscanning antenna systems. Radar at this frequency does not significantly penetrate foliage and the signal is unaffected by fog, low clouds, or normal rain. The Doppler shift of the return signal when detecting a moving target varies from a low audio tone (between 50 and $100 \mathrm{~Hz}$ ) for a walker to a high audio tone of several $\mathrm{kHz}$ for a fast vehicle. If the radar is scanning an area, these tones will occur in short bursts, and will be modulated by the PRF of the radar. The MSR has seven basic modes of operation as listed in the following table.

Table 1. Target Detection Modes

\begin{tabular}{|c|c|c|c|}
\hline M1 & 1 & 1200 & WM near range \\
\hline $\mathrm{M} 2$ & 1 & 2500 & $\mathrm{~V}$ near range \\
\hline M3 & 7 & 800 & WM mid range \\
\hline $\mathrm{M4}$ & 7 & 1000 & $\mathrm{~V}$ mid range \\
\hline M5 & 63 & 350 & WM far range \\
\hline M6 & 63 & 450 & $\bar{V}$ far range \\
\hline$\overline{\mathrm{M} 7}$ & 63 & 200 & High Sensitivity \\
\hline
\end{tabular}

WM $=$ Walking Man

$\mathrm{V}=$ Vehicle

near range $=<1 \mathrm{~km}$

mid-range $=1-3 \mathrm{~km}$

far range $=>3 \mathrm{~km}$ 
Pulse compression is a technique used by many radars to transmit longer pulses (i.e., more energy) to enhance long range detection while retaining the range resolution of the shorter, nonencoded pulses. The MSR uses bi-phase pulse coding. At near ranges, where a strong return signal is expected, only a single bit is needed, but at longer ranges. either a 7 bit or 63 bit code sequence is used. As a result, this radar obtains accurate range and velocity information from a walking man at ranges that we tested beyond 4700 meters, ind vehicles considerably further. The actual length of these code sequences depends on the range resolution selected. For 15 meters resolution, each code bit is $100 \mathrm{~ns}$ long, but $200 \mathrm{~ns}$ in length for 30 meters resolution.

The detection thresholds for this radar have default values as listed in Table 1 . However, after selecting the mode, the operator can increase or decrease the detection threshold depending on the nuisance alarm conditions and the expected target signature. The radar does have some automatic threshold adjustment capability to reduce excessive alarms from wind-blown vegetation, although it is not completely effective in areas with large bushes and trees moved by wind above $10 \mathrm{mph}$ or so.

\subsubsection{Target Range}

Range resolution is another user option. The returned signal is stored in 128 range bins. A bin can represent either 15 meters or 30 meters depending on the range resolution selected. At 15 meters resolution, the radar will cover a range interval of 1920 meters. Selecting the 30 meters resolution option produces a range interval of 3840 meters. The near range of the detection range interval is selectable from a minimum of 100 meters to a maximum of 8500 meters. Selection of the 30 meter resolution seemed to give less detection sensitivity, but we did not perform any tests to confirm this. All of our testing was done using the 15 meter range resolution setting.

\subsubsection{Target Velocity}

The velocity of the target toward (or away from) the radar is determined by measuring the Doppler shift of the radar carrier. The transmitted pulse at $9.4 \mathrm{GHz}$, when reflected off of a moving target, is shifted in frequency (Doppler shift). Once in the receiver, this shift in frequency is separated from the carrier and can be heard as an audio signal. Both inphase and quadrature phase audio signals are obtained, sampled and stored for each range interval. A digital signal processor is used to make a complex Fast Fourier Transform of these time signals into the frequency domain. The frequency spectrum consists of 128 discrete frequency bins. These bins represent 64 approaching and 64 receding target velocities. The highest velocity in $^{n}$ is about 30 $\mathrm{mph}$, the minimum, about $.5 \mathrm{mph}$. The MSR alarms only if a target has a radial velocity of at least $1.5 \mathrm{mph}$.

If a target such as a vehicle is approaching faster than $30 \mathrm{mph}(50 \mathrm{~km} / \mathrm{hr})$, then the indicated velocity will be less than 30 . This results from the PRF being 1789 pulses per second. These radar pulses act to sample the Doppler signal, and, as with any sampled data system, frequencies above the sampling rate are "folded" down and appear in the output as a lower frequency, or in this case, target velocity. 


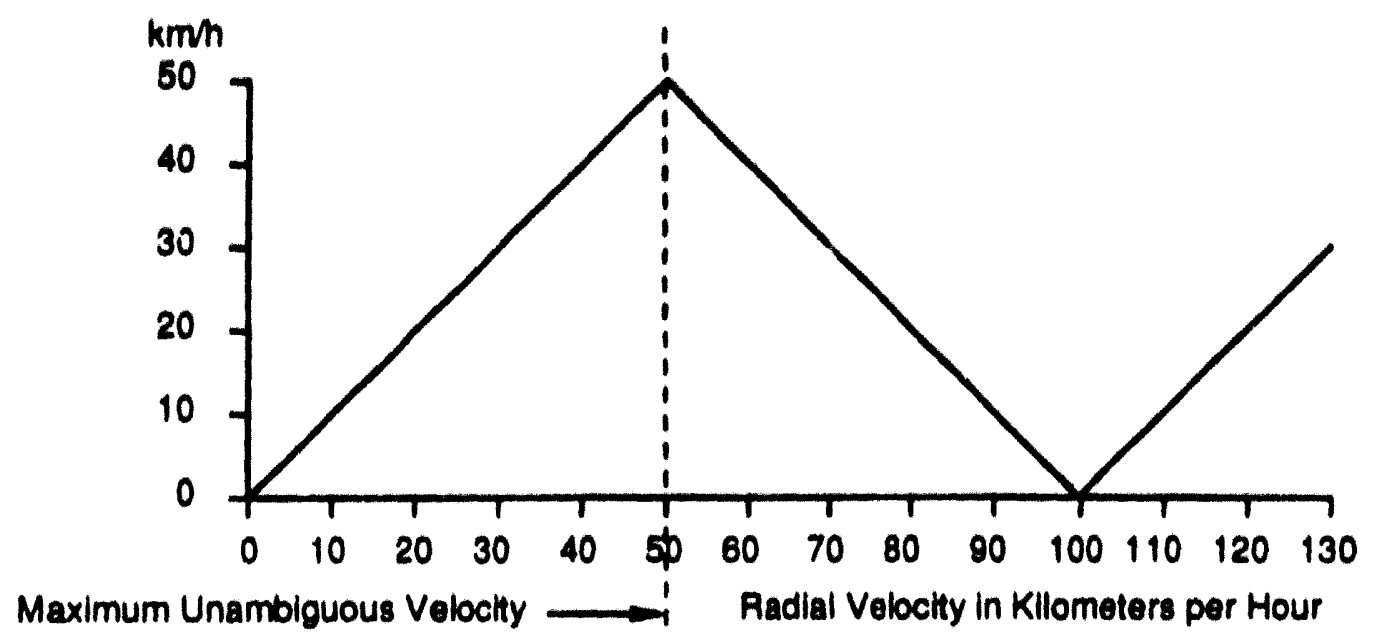

Figure 2. Velocity-Folding Diagram

\subsubsection{Scan Coverage}

The operator has the choice of three scan modes: $90^{\circ}, 30^{\circ}$, and dwell. During normal operation, radar returns are displayed on the screen with the antenna azimuth angle displayed horizontally. and target range displayed vertically. The operator may position a screen cursor over the target return blip in order to display the range, azimuth and radial velocity of the target on the screen. and to hear the Doppler audio through the headphones. The Doppler audio will not be heard unless the next return falls within the same range as the operator's cursor box.

The operator normally selects the $90^{\circ}$ scan mode to give the largest coverage. Once a possible intruder is detected, the operator may choose the $30^{\circ}$ scan to get more frequent return data. The antenna scans at a constant $8^{\circ}$ per second, so the $30^{\circ}$ scan angle will refresh the data three times faster, while still allowing considerable cross scan tracking. In the dwell mode, the operator will get a nearly continuous update on the target movement as long as the target remains within the antenna's beamwidth. Since cross-track movement cannot be detected, the operator must take care not to let the target move out of the $4.5^{\circ}$ radar beam.

\subsubsection{Antenna}

The MSR-20 comes with a slotted array antenna that has a horizontal and vertical beam width of $4.5^{\circ}$. Other beam widths may be appropriate for certain situations, and the pros and cons of these are discussed extensively in Section 4.8 .1 of this report.

\subsubsection{Performance}

Table 2 lists Motorola's performance claims for the MSR-20. The figures in this table are for a claimed $90 \%$ probability of detection in a single scan and a false alarm rate of one every 7.68 minutes. 
Tuble 2. MSk-20 Detection Specificution

(provided hy Mlotorola)'

\begin{tabular}{|c|c|c|}
\hline One Wilking Nian & $1 \mathrm{~m}$ & $x \mathrm{~km}$ \\
\hline Three Wilking Men & $3 m^{2}$ & $10 \mathrm{kml}$ \\
\hline Small Vehicle & $10 m^{2}$ & $19 \mathrm{~km}$ \\
\hline Targe Vehicle & $1\left(111 \mathrm{~m}^{2}\right.$ & $211 \mathrm{~km}$ \\
\hline Tlelicoplet & $10 m^{2}$ & $15 \mathrm{~km}$ \\
\hline
\end{tabular}

\subsection{Operation}

Alter selling up the $\Lambda R I$ on its tripod with the index mark pointed an the center af the desifed coverage area. the cables are connected. and the sy stem powered by a 24.28 Vide soure 1 lie

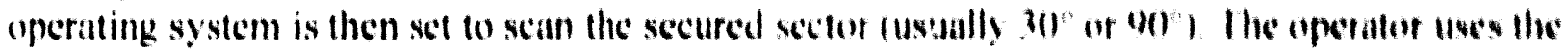
(D) / (Sec Figure 3.) fo select the operating modes, such as scan anple, range, runge tesolitionl. and threshold. The ('D) I displays target returns of "hits" on a "map" of tante (lentical avis) azimuth angle (horizontal axis). The operator can obtain target velocily data, and newe persiase range and angle data by moving a cursor over a display "hit".

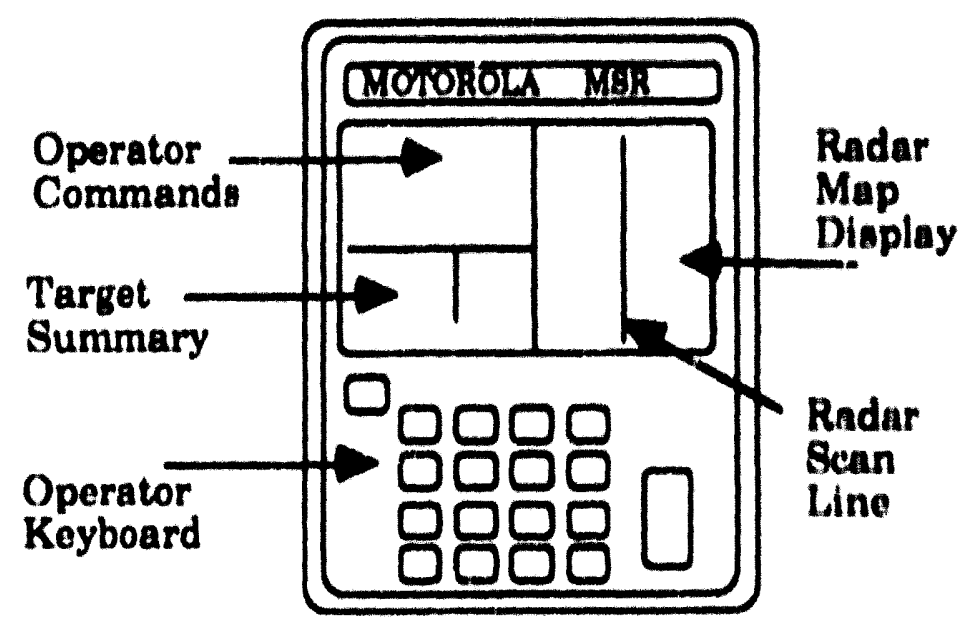

Figure 3. Control and Dixpluy I nit

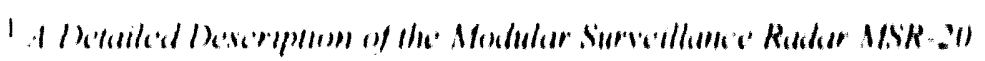

Motorola, Inc., Iactical Iilecironics Division. |40) 
The detection mode can be set for the distance and kind of targets expected. If, for example, walking infiltrators are expected, and the nuisance alarm rate is inw, then a more sensitive mode is selected If only vehicles are to be detected, less sensitive modes are used.

The operator will normally activate the alarm beeper if he is not watching the screen. When the alarm sounds, the operator moves the cursor box over the alarm spots on the user screen, then reads the summary information display. This display provides target radial velocity, azimuth location, and indicates whether the target is advancing or receding.

This information can be passed on to the response team. If more information (target assessment) is required. the radar can be put into the dwell mode and moved to the target azimuth to get better Doppler audio signals, range, and velocity information. If the range is within 1000-2000 meters, the operutor or a companion may use binoculars, night vision goggles, or a thermal imager to get a heller assessment. While the radar will continue to detect well at longer ranges, assessment will be limited to interpretation of the radar return signals.

\subsection{Performance}

The testing done during this evaluation had two major thrusts:

I II conlirm the advanced performance of the MSR-20, and

2. II identify those conditions that would adversely affect the usefulness of this radar in DOE applications.

Motorola states that this radar will detect a single walking human $\left(1 \mathrm{~m}^{2}\right.$ radar cross section) at 8 $\mathrm{km}$. We were able to confirm detection out to nearly $6 \mathrm{~km}$, and have no reason to doubt the $8 \mathrm{~km}$ ligure umder ideal conditions. Of course. vehicles can be detected at a greater distance. The manufacturer indicates that small vehicles with a $10 \mathrm{~m}^{2}$ radar cross section (RCS) can be detected out to $15 \mathrm{~km}$, and large vehicles with a $100 \mathrm{~m}^{2} \mathrm{RCS}$ can be detected out to $20 \mathrm{~km}$ under ideal conditions. We did not attempt to confirm these ranges. Such ranges are not easy to confirm in the Nlbuquerque area and are of little application to the normal DOE site security problem. except possibly at NTS.

Moforola project personnel tell us that the detection algorithm in this radar is designed to fencrate only one false alarm in 7.68 minutes. We found that nuisance alarms or other unknown returns generally gave much higher alarm rates.

Motorola specifies a minimum detectable target velocity of $.6 \mathrm{~m} / \mathrm{sec}$, about $1.4 \mathrm{mph}$, and states that it can detect a crawling individual at close range. We found that a walking man could be moving at a considerable angle to the radar $\left(60^{\circ}\right)$, and still be detected, especially at the closer ranges of interest (under $2 \mathrm{~km}$ ). This tends to support Motorola's minimum velocity specification since a walking man will often move at about $1.5 \mathrm{~m} / \mathrm{s}(3.5 \mathrm{mph})$. At a $60^{\circ}$ angle, the corresponding radial velocity is $0.75 \mathrm{~m} / \mathrm{s}$, slightly higher than the $0.6 \mathrm{~m} / \mathrm{s}$ specification. We found that the false alarm rate due to blowing grass and brush was acceptable when the threshold was sel to detect intruders at ranges less than $2 \mathrm{~km}$, and normal wind conditions $(<8-10 \mathrm{mph})$ existed. 


\section{Testing}

\subsection{Test Constraints}

We found that several characteristics of this radar required us to pick the test area carefully and to limit the test range. The radar will respond to moving vehicles at ranges closer than those selected, apparently through double reflections to targets that are outside the area of interest. The worst offenders are large flat-sided metal trucks or vans. We found that if the radar is mounted high enough, local traffic in the near foreground is not a problem.

To distinguish true alarms from nuisance alarms due to blowing grass and small game, we selected times and areas that would minimize these effects. In our tests, moving targets located beyond the selected range gate caused no alarms.

\subsection{Test Areas}

Tests were conducted at Sandia's Exterior Sensor Lab, Robotic Vehicle Range, and at the Coyote Canyon Test area. These areas, located just south of Albuquerque on the Kirtland military reservation, are shown in Figures 4 and 5. The Exterior Sensor Lab and Robotic Vehicle range are on flat, grass-covered land. It is normally free of,-human activity, and an observation tower at the RVR provides good test control. For the Coyote Canyon test area, the test range was much smaller, a gently sloping area that has a vegetation zone transition from desert grass to a piñon forest. The purpose of the Coyote Canyon tests were to determine radar penetration of sparse vegetation and a denser tree line.

\subsection{Test Targets}

Most testing involved a single human target having a significant radial velocity (greater than $.6 \mathrm{~m} / \mathrm{s}$ ). Tests of vehicles invariably showed a higher return than for a single human. It became apparent that approaching vehicles have such a large radar cross section that the detection threshold could be raised enough to prevent nuisance alarms from wind blown brush or small game and still provide reliable target detection. For this reason, additional vehicle testing was not included in this study.

\subsection{Summary of Test Results}

\subsubsection{Test Series I (3900 Meters)}

The first tests were run in late June with the radar mounted on the roof of the Exterior Sensor Laboratory (17' above the ground), and pointing across Pennsylvania Blvd. toward the NE. The target walked radially at a range of $3.9 \mathrm{~km}$ with the range interval set from 2500 to 4420 meters. The target function was set to mode 7 , and range resolution to 15 meters. To cut down returns from activity in adjacent areas, the radar was operated in the dwell mode, covering only the area shown in Figure 4. Testing started at 6:20 in the morning in clear, calm weather. The normal threshold for this mode is 200 . At this setting we got good detection for a walking man (WM), about 40 alarms per minute. However, we were also getting nuisance alarms. These were not only from medium to large trucks on Pennsylvania Blvd., but also (we think) from side lobe and near field effects from small trucks and vans driving nearby about 100 meters away from the radar. The Pennsylvania traffic was at a range of only 1700 meters, and should have been rejected by the selected range gate. (We have since speculated that this effect may be due to reflections of landscape features from the moving, flat sided, trucks.) 


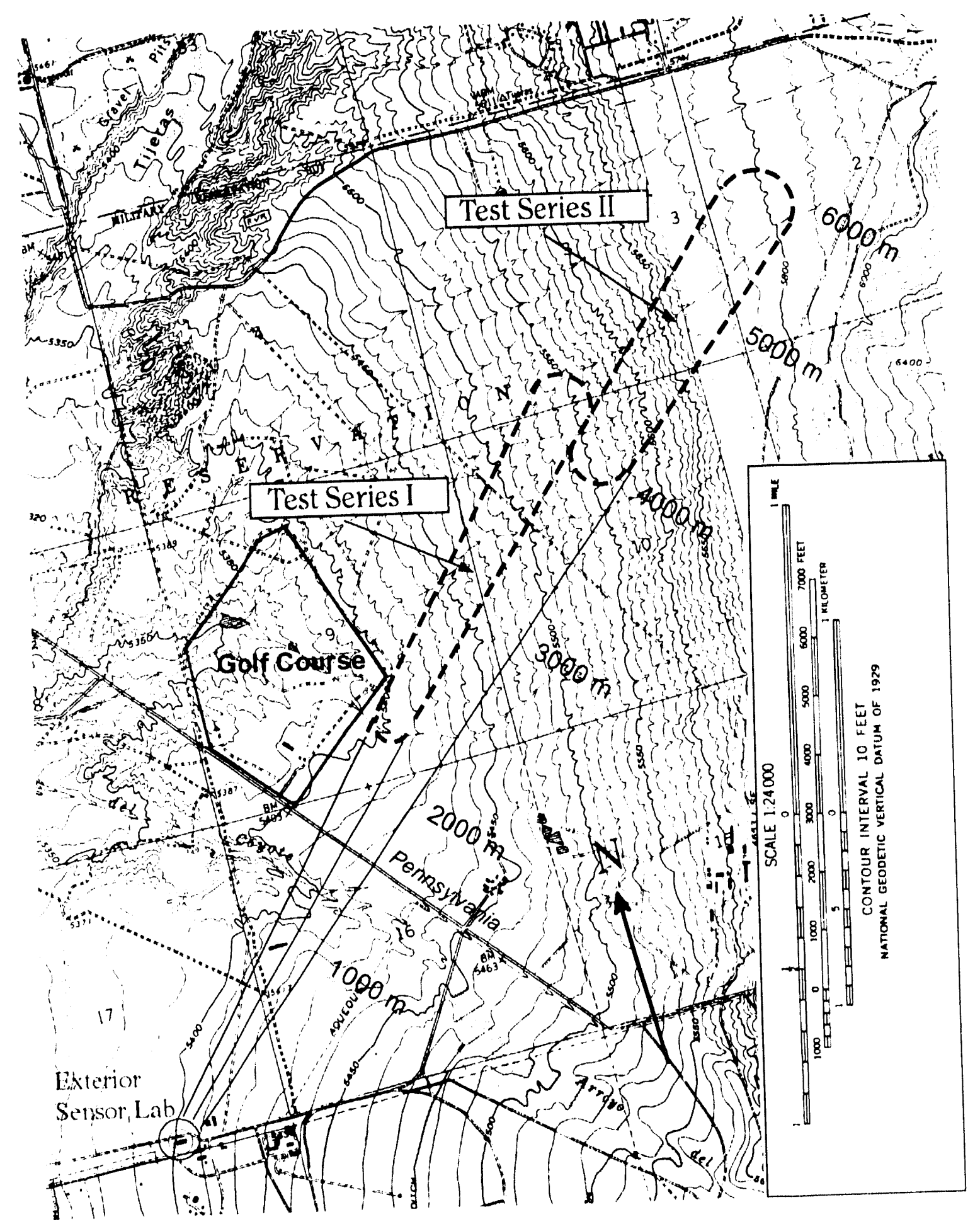

Figure 4. Exterior Sensor Lab. Range, Test Series I \& II 


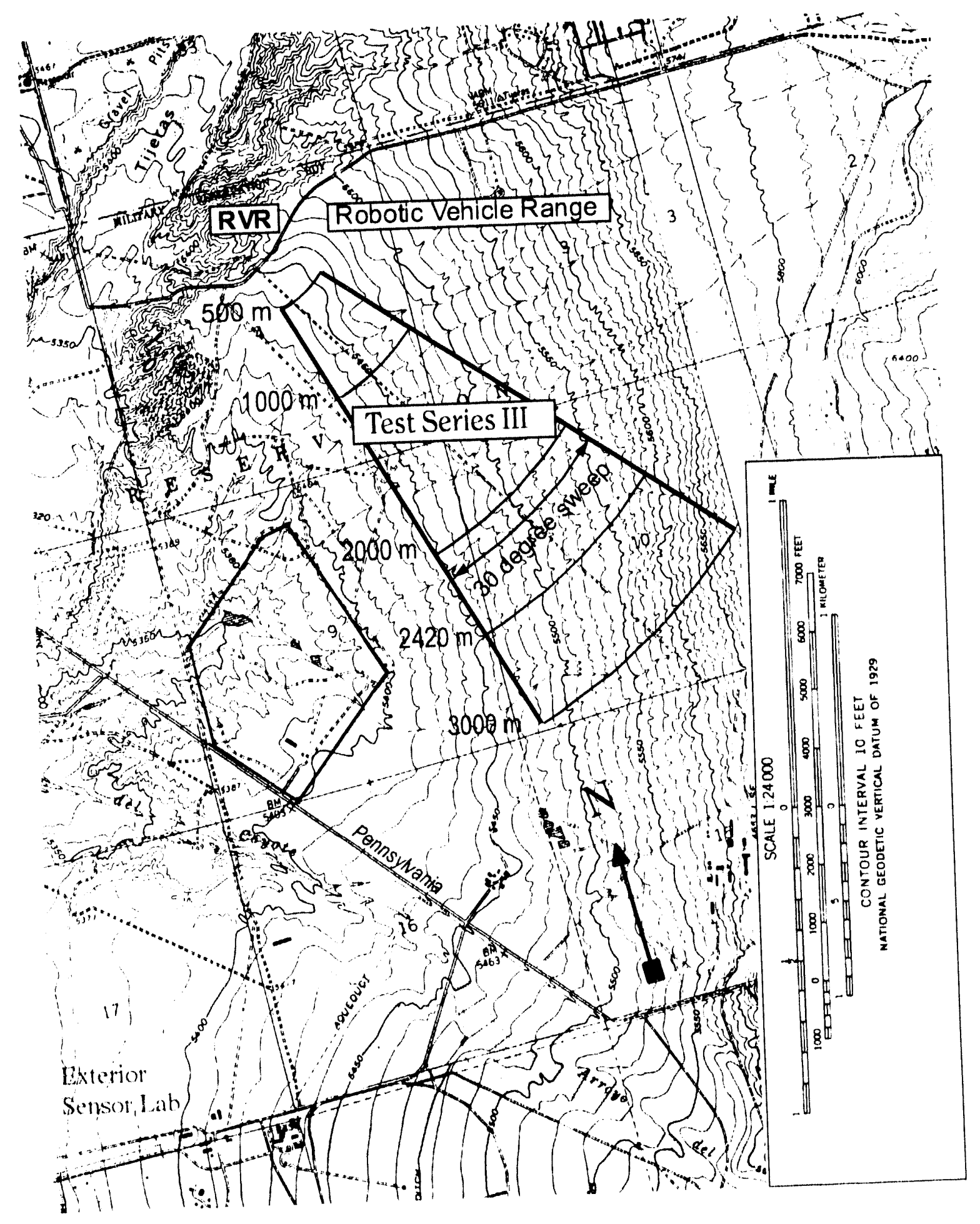

Figure 5. Robotic Vehicle Range, Test Series III 
As the threshold was lowered to 150 and 100 (from the normal mode 7 value of 200), the false/nuisance alarms increased, and the number of true alarms decreased. There was no information displayed from alarms at these thresholds. Higher thresholds $(300,400,500)$, produced fewer valid detections, but did give valid summary data information. The number of false/nuisance alarms also fell dramatically. A threshold setting of 400 would have been a good compromise for this situation, but could only have been arrived at empirically. In summary, the default threshold value of 200 was found to be satisfactory, but for this particular situation a threshold of 400 allowed for adequate detection while lowering the NAR. Operator adjustment of the thresholds should not be expected for DOE application of this radar.

\subsubsection{Test Series II (4600 Meters)}

The next test series (early morning on July 1) was similar to the first, but the range gate was set from 4000 meters to 5920 meters. The default threshold was 200 , and we again operated in mode 7 . Vehicles within 60 meters of the radar were still a problem; however, the trucks at 1700 meters gave almost no false alarms. The walking target (at 4645 meters) gave an alarm rate of 20 per minute at the default threshold of 200. A setting of 300 also gave good detection of a walking man and no false alarms. A threshold of 400 gave almost no true alarms nor any false alarms.

\subsubsection{Test Series III (2600 Meters)}

For this series, we moved all of the equipment to a pair of test towers at the Sandia Robotic Vehicle Range (see Figure 6). This gave the advantage of a "cleaner" (albeit shorter) range, higher mounting for the radar, and the longer focal length zoom lens for the assessment cameras (see Figure 7).

The radar was mounted on the handrail of the old guard tower (right hand tower in figure 6). Test control and alarm assessment used the thermal IR and video cameras mounted on the left hand scaffold tower. This range normally has no moving vehicles or personnel, and only a few animals (coyotes) during the day. Figure 8 shows the view of this range from the radar. The visible light and IR assessment cameras (shown in Figure 7) had good visibility and could be panned to the radar detection azimuth.

For the next test, the range interval was set at 1000 to 2920 meters, and the target (TGT) control was set to mode 3. Our walking man was placed at 2665 meters. Range resolution stayed at 15 meters. Because of the lack of other range area activity, the 30 degree scan could be used in place of the dwell mode used before.

As in the previous series, we neasured the false and true alarm rate as a function of threshold value to determine if the default value was best for this situation. We found that it was. At thresholds of 800 (default value) and 1000 we got 6-8 true alarms per minute and no false alarms. As the threshold was increased, the true alarms decreased until there were no detections at all for a threshold of 2500 . As the threshold was decreased below 600, the false alarm rate (FAR) increased rapidly, to 7 per minute at 400 .

For the next test, a walking man at 1600 meters gave 10 true alarms per minute and no false alarms were recorded. At a threshold of 1500 , there were still 6 true detections per minute, dropping to 1 per minute at 2500. There were no false alarms until the threshold was dropped to 400 . At this threshold there were about 7 per minute. The range interval used for this and the next test was 500 to 2420 meters. 


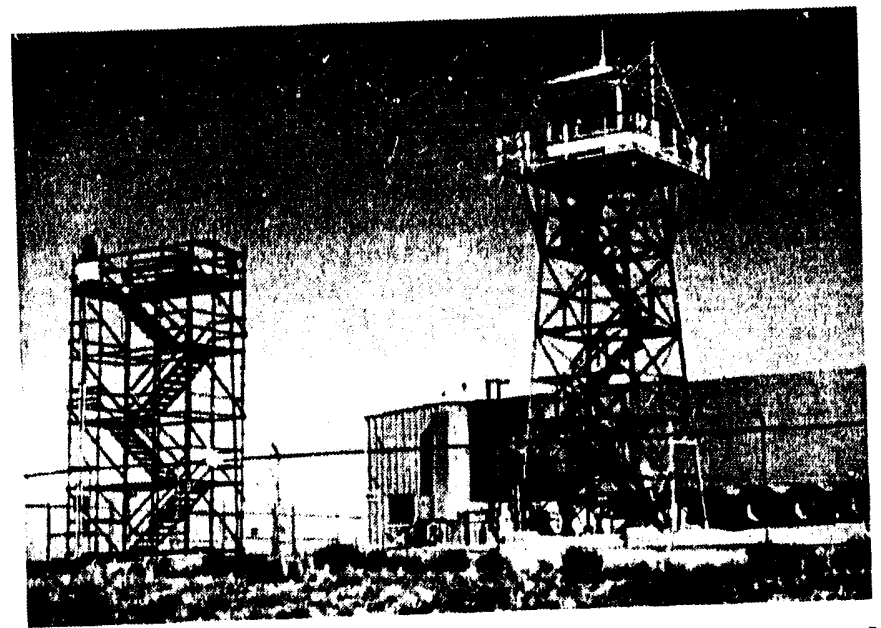

Figure 6. Test Towers Used in Testing the MSR-20

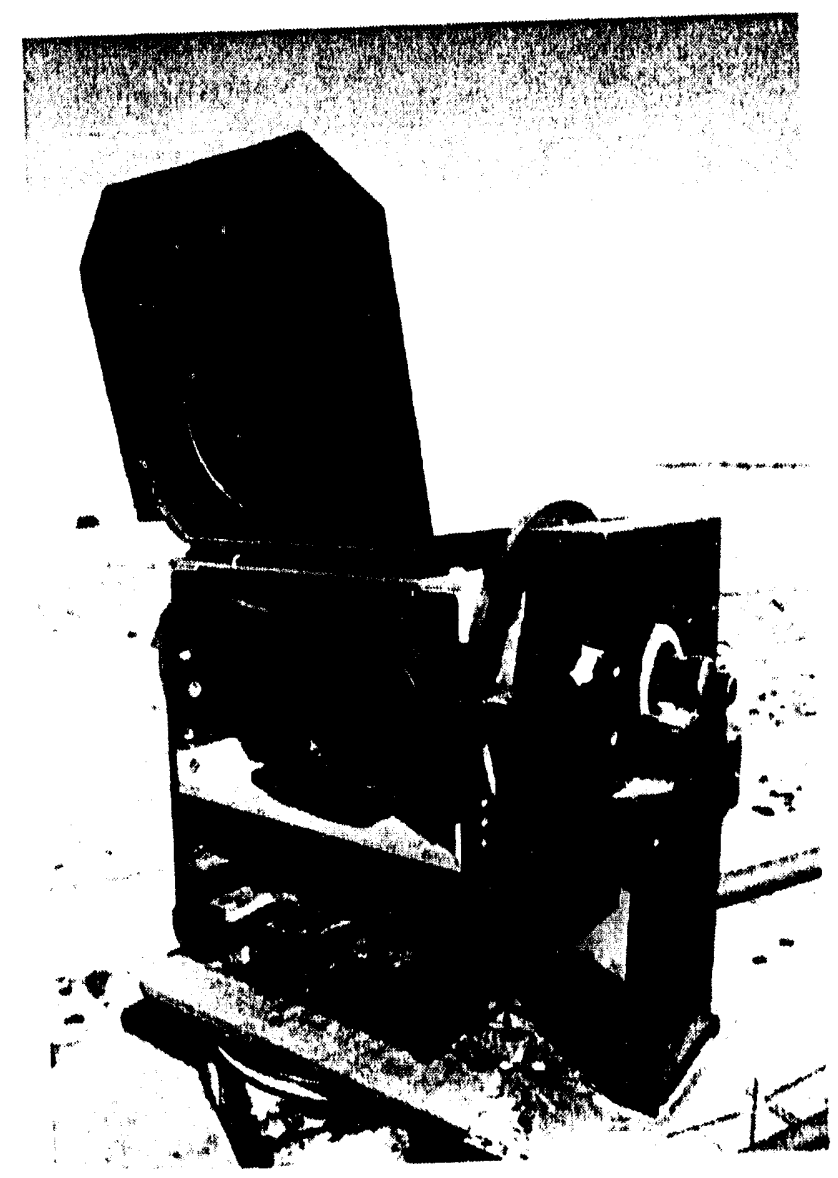

Figure 7. CCD Camera and FLIR 


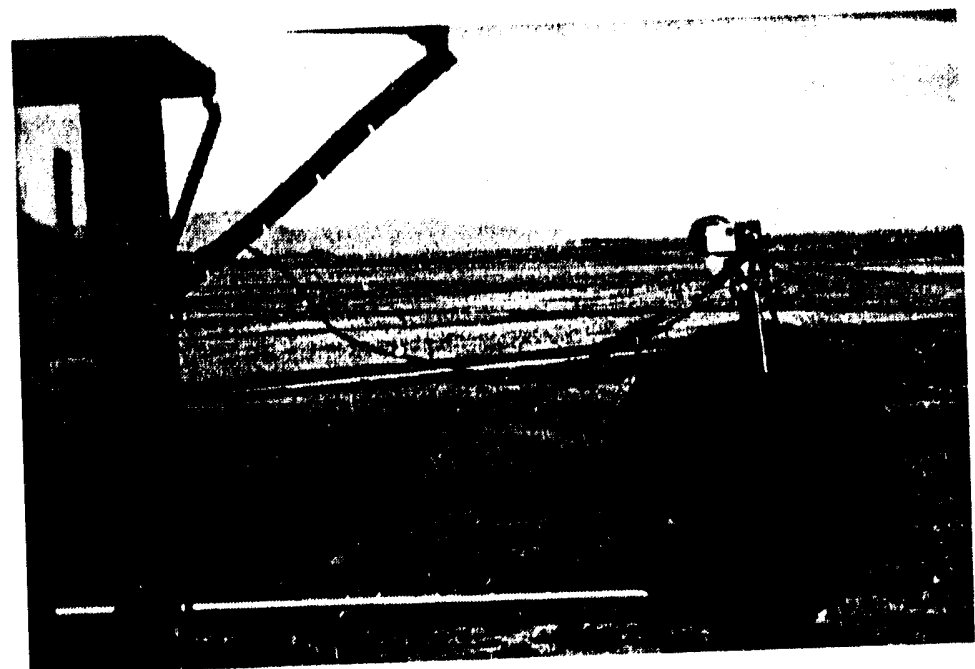

Figure 8. MSR-20 Looking South from the Guard Tower-Robotic Vehicle Range

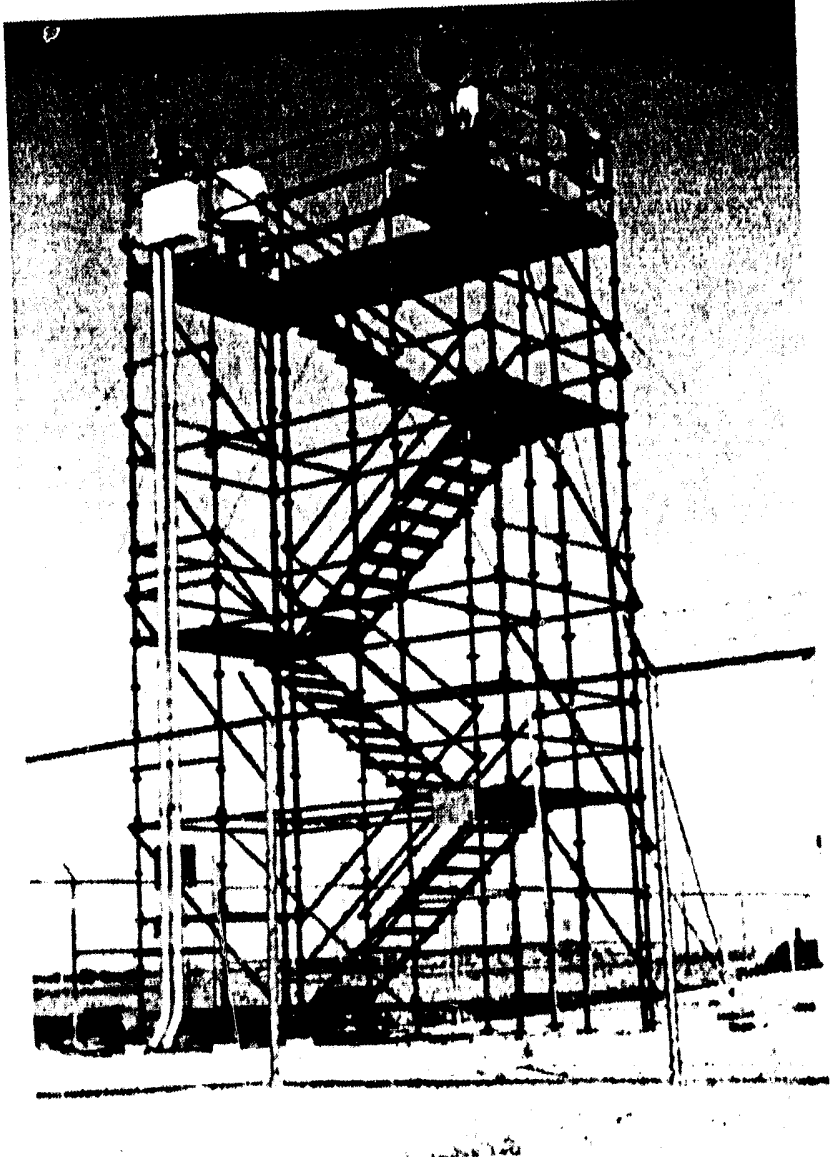

Figure 9. Test Tower and Radar 
In the last test, the target walking at 650 meters generated about the same number of true alarms per minute (8) at a threshold of 800 . At a threshold of 1500 , there were only 5 per minute, and none at all at 2500. Since all radar settings were the same as before, no new FAR data was recorded.

The data from all three of these tests have been plotted together in Figure 10. The lower trace represents the number of false or nuisance alarms per minute. The radar was set for a $30^{\circ}$ scan angle and at ranges from 500 meters to 2920 meters. Note that the system was essentially free of false alarms at any threshold over 600 . The upper trace is the rate of combined true and false alarms, which is the same as the true detection rate at thresholds over 600 under these very favorable conditions. This true detection rate seems low until one realizes that, with a $30^{\circ}$ scan, the radar is only "seeing" the target once every 4 seconds or 16 times per minute.

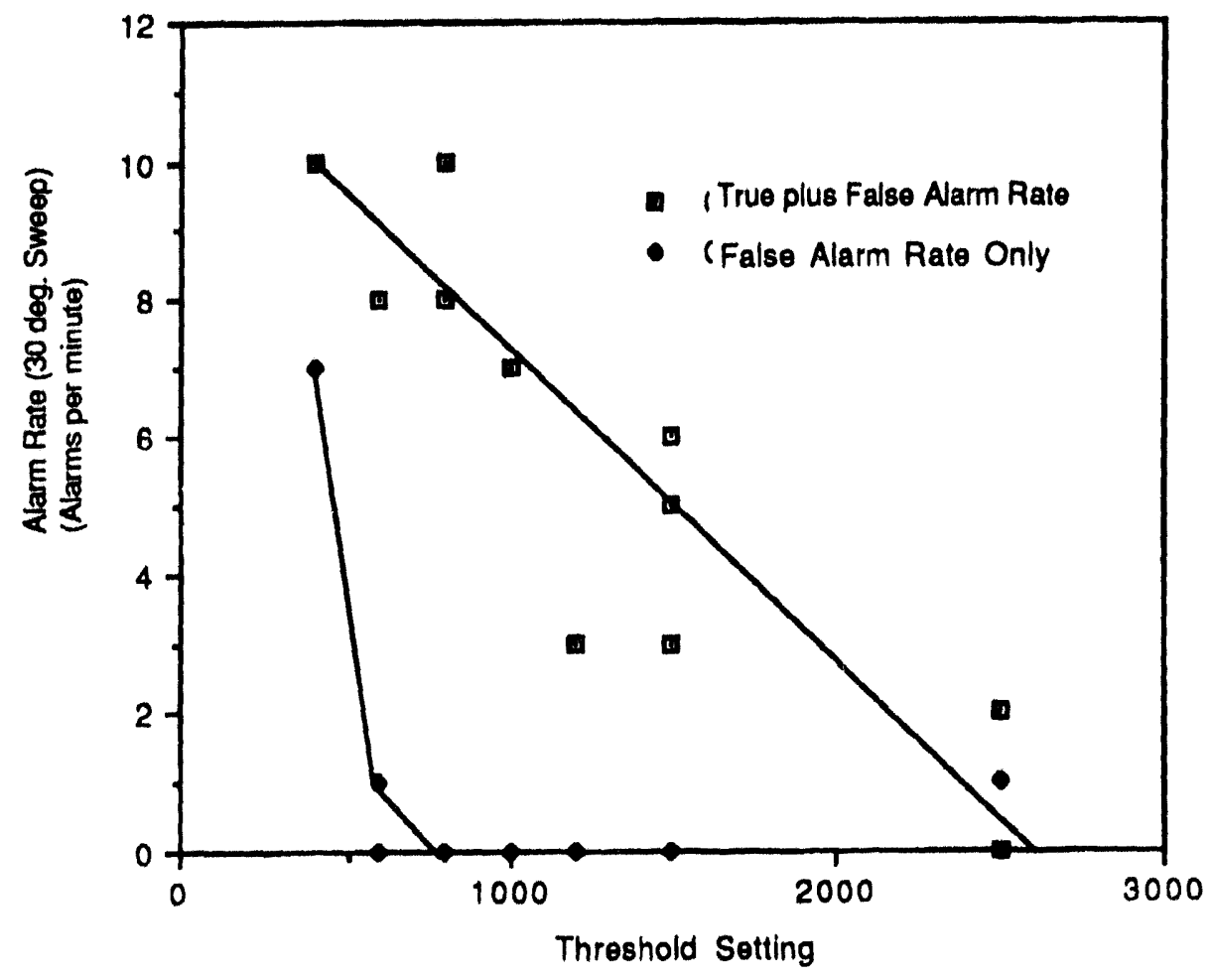

Figure 10. Plot of True and False Alarm Rates Vs. Threshold (Combined Data from Tests) 


\subsubsection{Test Series IV (550 Meters in Light Brush)}

The purpose of this test was to determine if this radar could detect a person approaching from behind heavy, medium, or very light brush. The test was conducted in Coyote Canyon, in early August, on the west side of the Manzano mountains on the Kirtland AFB reservation (see Figure 12). The radar was set up on the ground near the intersection of No Sweat Blvd. and Coyote Springs Road, at the mouth of Madera Canyon. The area covered had desert grass and low cactus, with scattered tall, thin cactus (Buckhorn Cholla) for the first 600 meters. At 700 to 800 meters, dense piñon bushes grow to form an uneven tree line. The target walked in a small elliptical circle with the major axis ( 1 meter) in line with the radar. This was an attempt to simulate the small motions of a terrorist who may be setting up equipment or moving to get a better view of his intended target (see Figure 11). When we found that an intruder moving in this manner behind the 6 tall cholla could not be detected, the target walked a greater distance, about 6 meters, but was still not detected. One could easily see through this cactus, and an intruder behind such a plant could easily observe his target and prepare for the launch of a stand-off weapon undetected by this radar. Of course, we found that detection of intruders behind any of the medium or dense bushes was not possible either. We noticed also that wind gusts estimated at 15-20 mph caused alarms from the wind-blown bushes and trees.
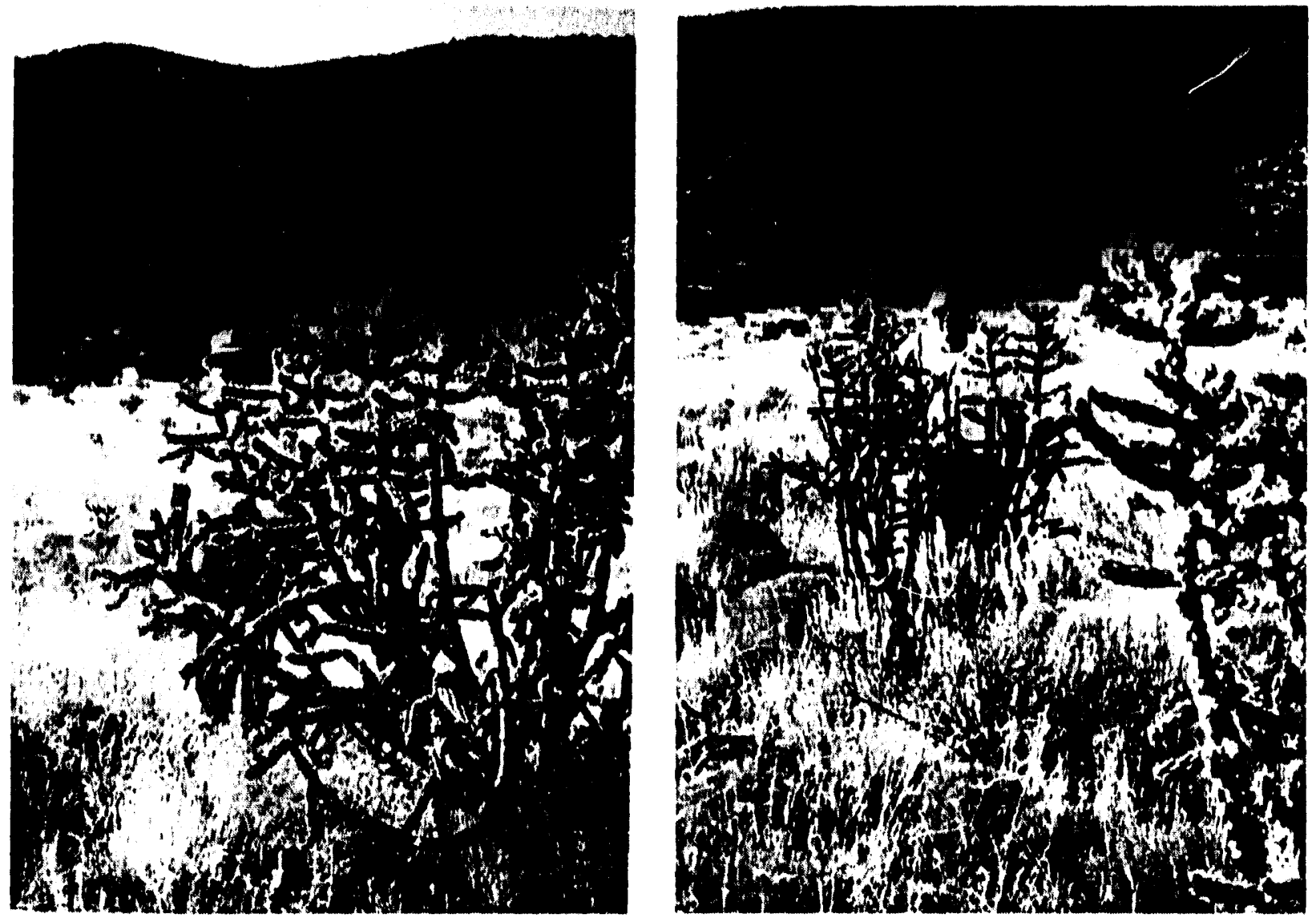

Figure 11. Human Target Hidden by Cactus 


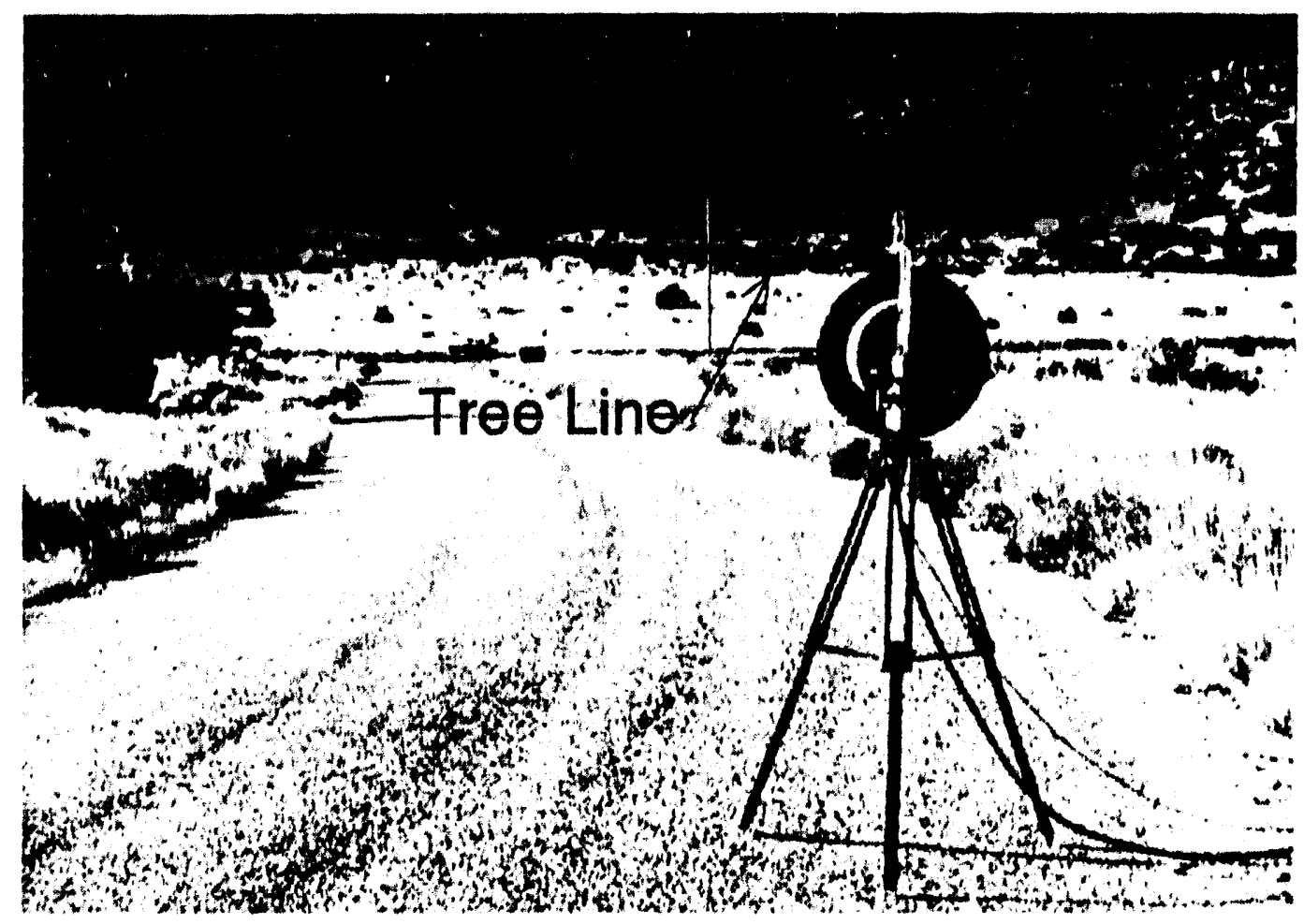

Figure 12. Radar Set I p to Defect Personnel in Tree line
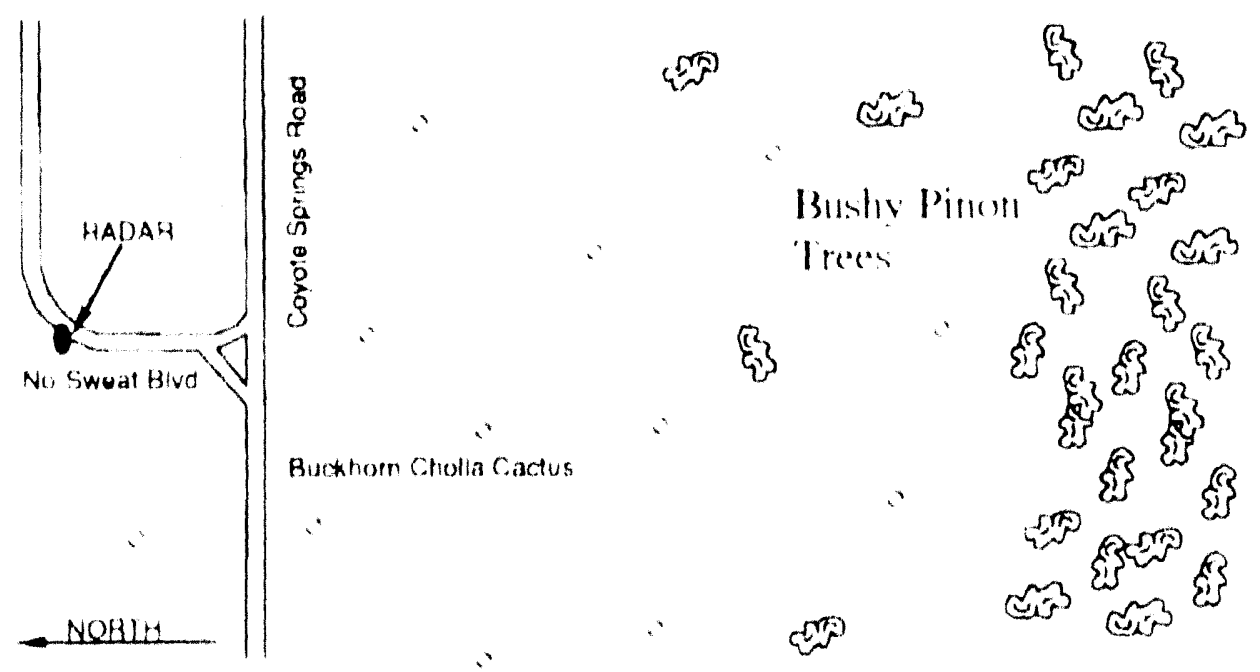

Figure 13. The Radar Test Range al conote Cangon

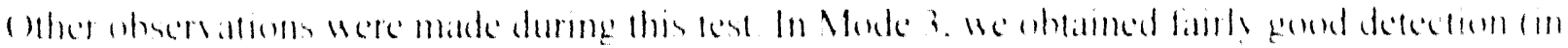

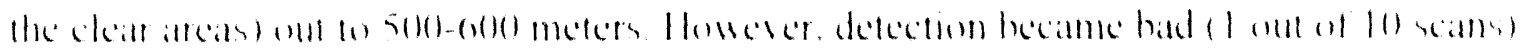

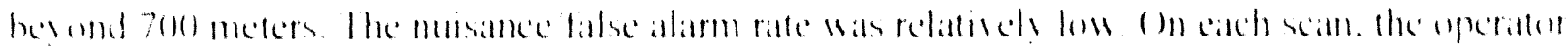

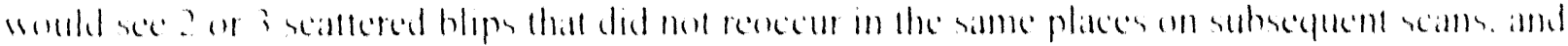

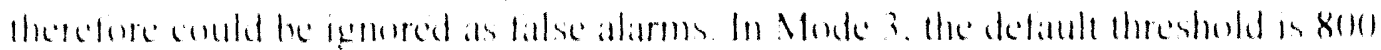


When we switched to Mode 1 to increase detectability, we experienced bad nuisance alarm rates or false alarm rates even in relatively low wind. However, this mode did allow detection out to about 900 meters. Detection of a walker was sporadic; it was hard to tell the nuisance and false alarms from the true alarms. When Mode 1 was used on the walker at 600 meters, we got multiple detections, with the alarm summary indicating both approaching and receding directions. Specifically, a single walker would be reported by the radar as 2 or 3 approaching targets and 1 or 2 receding ones. In this mode, the threshold was 1200 . We concluded that under breezy conditions with tall vegetation, the radar may not be useful.

\subsubsection{Assessment Test (Series V)}

\subsubsection{Human Intruder Assessment}

For these tests, the radar was mounted on the scaffold tower as shown in Figure 9. Early morning tests on July 2 were conducted to evaluate the use of a thermal imager and a long focal length camera for the assessment of targets picked up by the radar. The thermal imager had dual horizontal fields-of-view of $3^{\circ}$ and $10^{\circ}$, and the CCD zoom lens horizontal coverage was from $2.5^{\circ}$ up to $25^{\circ}$. The thermal imager and camera were mounted on a common pan/tilt platform and are boresighted. We measured the angular offset between the radar azimuth readout and the VISDTA pan and tilt azimuth indication. This allowed us to reliably locate the intruder using the radar information once the camera elevation was properly set. For an actual installation, the radar azimuth readings must be well calibrated, and there should be a look-up-table that will correlate radar range readings to expected elevation settings of the camera pan and tilt mount.

With those problems resolved, target contrast and movement were the next hurdles. At the RVR early in the morning, the target is front-lit and front-warmed by the sun, and generally gives outstanaing contrast in a thermal imager. However, if beyond 1500 meters, assessment was difficult without some noticeable lateral movement. (Frame flashing works well here.) We were generally satisfied with this assessment technique for ranges out to 2000 meters, but finding an intruder beyond this can be difficult, except under ideal conditions. We found that the MSR sometimes reported azimuth readings with as much as $4^{\circ}$ of error, depending on the scan direction. This may have been due to a loose potentiometer. This error had to be added to the $4.5^{\circ}$ beam width, in figuring the azimuth for the assessment cameras.

\subsubsection{Fast Truck Assessment}

This test was conducted to see if the operator could assess that a fast moving target was a vehicle and bring a CCD or IR camera to bear on it. In each trial, we started with the assessment cameras pointed about $90^{\circ}$ away from the expected target position. After a few trials, we found that it took approximately 20 seconds to get the radar cursor on a target blip and read the velocity and the azimuth. Then it took another 55 seconds to find the truck with the cameras.

For these tests, the truck was driving at approximately $24 \mathrm{mph}(11 \mathrm{~m} / \mathrm{s})$ according to the radar display. This speed was confirmed by the truck speedometer. Since the truck first appeared at 2000 meters, it had advanced to 1175 meters by assessment time. Another 1.5 minutes remained for security reaction. We conclude that the cameras, if needed for assessment, should be mounted on a fast pan and tilt that is slaved to the radar display cursor. 
During this test we observed that there was usually no audible Doppler tone. This is because the cursor must be set to the same range as the target while the antenna illuminates it before the Doppler tone can be heard. Also, the Doppler tone caninot be heard if the alarm beeper is "on".

\subsubsection{Performance Vs. Antenna Height Above Ground (Series VI)}

In general, a ground search radar's performance is expected to deteriorate as the antenna height above the ground decreases; multipath effects are more likely at shallow grazing angles and it is harder to maintain line-of-sight to the intruder, especially his legs. We believe that multipath played a major role in several areas relatively close to the RVR with good line-of-sight properties where detection was inconsistent for no apparent reason.

In this test sequence, we operated the MSR with the antenna assembly located at approximately $9 \mathrm{~m}, 6.5$ meters, and 2.5 meters above the ground on the RVR scaffold test tower. The target in all three trials was a walking human at a range of 1800 meters. We expected that the frequency of detection would decrease as the radar antenna was lowered from the top of the tower to the two lower platforms. Instead, we found that the percentage of scans where there was a detection actually increased from about $54 \%$ to around $88 \%$ when the radar was lowered from 9 meters to 6.5 meters, then decreased (as expected) to approximately $82 \%$ when the antenna was lowered to the 2.5 meters level (see Figure 14).

Although much more testing would be required to draw firm conclusions, these results suggest that the target may have been located in an interference zone for the 9 meters elevation, but when the antenna was lowered, none of the new interference zones coincided with the target, and sensitivity returned to normal.

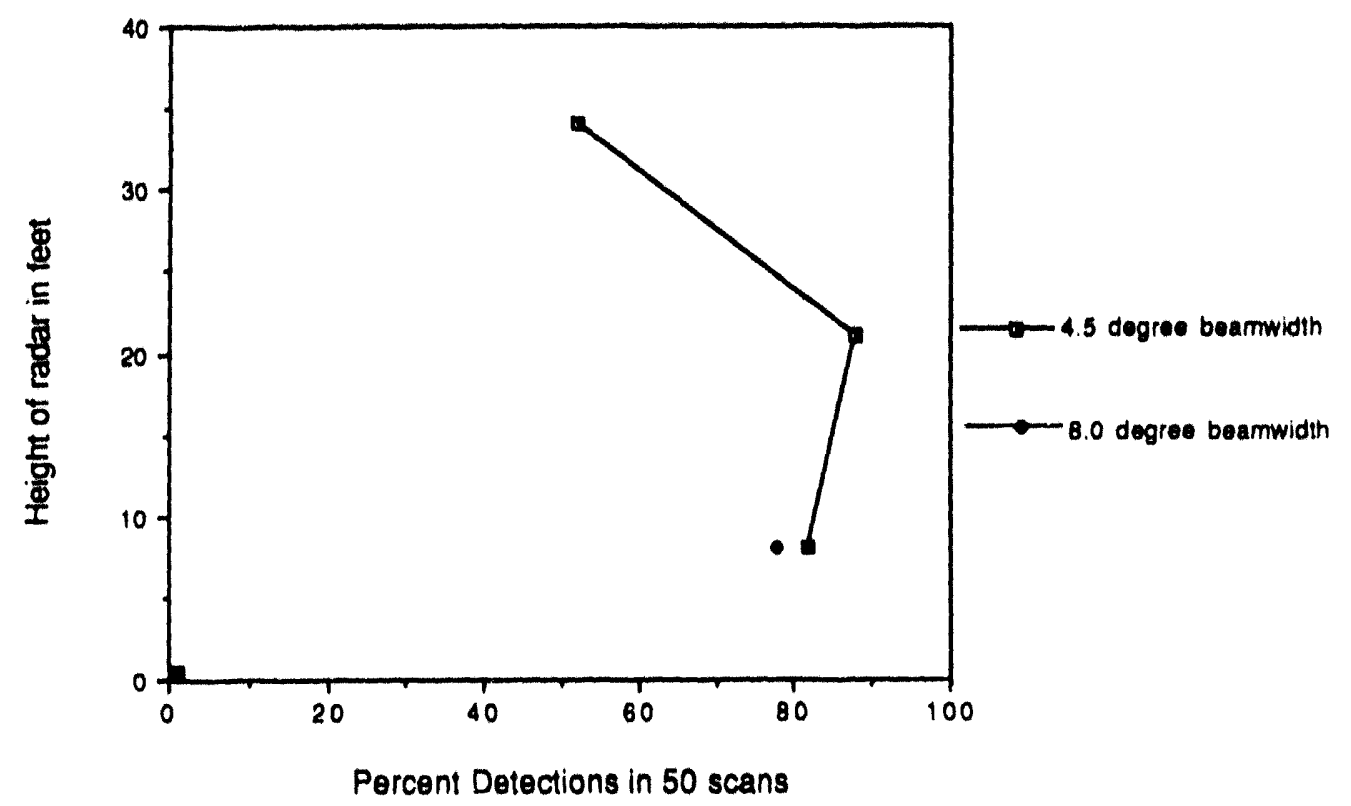

Figure 14. Plot of Percent Detections Vs Antenna Height at the RVR 


\subsubsection{Performance Vs. Antenna Beamwidth (Series VII)}

Since the standard $4.5^{\circ} \times 4.5^{\circ}(45-\mathrm{cm}$ diameter) antenna had yielded detection ranges far in excess of those we consider practical for DOE applications, we performed a brief test using the $8^{\circ} \times 8^{\circ}$ beamwidth $(30 \mathrm{~cm})$ antenna to verify that performance did not deteriorate to an unacceptable level at ranges of up to $2 \mathrm{~km}$.

For this test, after replacing the standard antenna with the smaller (wider beamwidth) variant, we repeated the last test that we performed using the $4.5^{\circ}$ antenna; we placed the ART at the 2.5 meters level and used a walking human as the target at the 1800 meters range we had used previously. The wider beamwidth resulted in a only a small decrease in detection probability, from $82 \%$ to about $78 \%$ of the scans.

This, admittedly limited, test tends to support our belief that the $30 \mathrm{~cm}$ antenna provides satisfactory detection, while allowing for less sensitivity to vertical tilt (easing equipment setup) and providing better coverage of upsloping terrain. The wider beamwidth may also permit a faster scan rate than the current $8^{\circ}$ per second. Two drawbacks to wider beamwidths are that they cause a larger uncertainty in the azimuth position report of the radar and a shorter detection range. The advantages and disadvantages of different beamwidths are discussed in more detail in section 4.8.1.

\subsection{Operator Observations}

The following observations were made when using this radar as a stand alone system. If it were modified to work as a sensor for a central alarm system, many of the following comments would not apply:

- The system detects walking humans and approaching vehicles at the default settings very well. We know that it detects wind-blown bushes and trees and it is conceivable that it will also detect shorter vegetation such as tall grass when moved by the wind.

- It can detect humans and vehicles at ranges too Sar for practical assessment with video cameras and thermal imagers. Very long focal length lenses can be used. but these can be very expensive. Also, the imprecise radar azimuth data would require the operator to scan a large angle in order to visually acquire the target. The MSR azimuth angle uncertainty is several degrees to either side of the displayed angle, while long FL lenses may have only $1^{\circ}$ of azimuth coverage.

- The system is difficult to learn because of the condensed keyboard. There should not be two different uses for the same key, and the labeling should be less cryptic. A new operator may have trouble using this radar, especially in a panic situation. Even experienced operators may forget how to access seldom-used functions.

- The Liquid Crystal Display (LCD) is hard to read, and the layout is distorted because of the use of angle instead of cross-range location for the horizontal axis (see Figure 15). In the military, this radar is used to direct gun fire, so azimuth is appropriate. For DOE security, it would be used to direct a response team to sector Cartesian coordinates. A conversion would have to be done either by the operator or by the central alarm map display computer. 


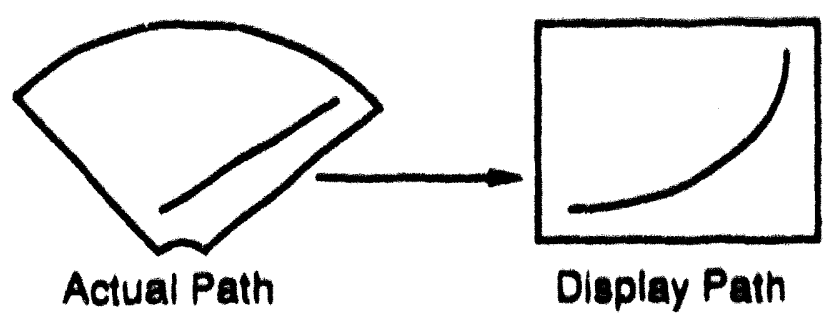

Figure 15. Distortion of the Intruder's Puth

- In dwell mode, you must have the cursor in place before the alurm is heard in order to yet alarm summary information. You cannot move it over the alarm point later as you can in sweep mode.

- To erase alarm points in dwell mode, turn "track" off, then hack on.

- The radar did not seem to hold its zero angle reference positio from one test to the next. This was unexpected: Motorola suspects there was a loose petentiometer or mountiny bracket.

- When in dwell mode, it was difficult to get the radar to point to a precise hearing. This was probably noticeable only because we had mounted a "bore-sighted" video camera to the antenna. Because of the width of the antenna beam, this lack of precision might not be noticed in the radar data or performance. This radar uses a potentiometer that is read only at the ends of the scans, or while the radar is at rest during the dwell mode. When the operntor calls for a small movement, the nicroprocessor furns the motor on for a short line. Becalise of gear lash. etc., the amount of movement that results is not precise.

- Small, but abrupt external motion of the radar set caused a lot of false alarms. This is probably because, from the radar's point of view, the whole world appeared io move.

- There were occasions when we heard an alarm beep, but no dot appeared un the screen. (H) ther circumstances would cause an alarm and a dot, but no summary information was available when the cursor was used. Motorola had not experienced this phenomena, and had no explanation.

- The Doppler shift audio may he a good assessment tool for distinguishing vehicles from humans. It may not be as good for distinguishing humans from unimals. Reading the velocily given in the summary data readout seemed to be more useful and consistent than using the Doppler to determine the velocity of a target. We found that the cursor must already be where the target blip will appear in order to hear the Doppler audio when the radar is scanning.

- The RES (Resolution) button provides for operation in either a 15 or 30 meter mode. While the 30 meter resolution sacrifices the ability to detect individual targets. if increases the range coverage interval from 1920 meters to 3840 meters. (iond range resolution is most importani where the radar is used for assessment as well as detection. and less important if the required range is close enough ( 2500 meters) that assessment eameras can be used. We did not levt io confirm detection of a walking target when using the 30 meter resolution mode. 
- A resolution of 30 meters in both the dwell and scan modes caused a constant high-pitched whine in Modes 5-7. A resolution of 15 in both the dwell and scan modes caused static in Modes 1-4 and a soft, high-pitched sound in Modes 5-7. These sounds greatly interfered with the operators ability to assess the audio Doppler shift, and to respond appropriately to the alert tone. We do not know if this affects the automatic radar detection.

- Because the display shows down-range distance, but cross range angle, the motion of the target appears distorted for close targets to a person used to looking at a normal Cartesian map display. It is mentally tricky to compare motion at 2500 meters with similar motion at 500 meters because of the angular vs. linear display (See Figure 15 above).

- The CDU and SPU must be turned on in a prescribed sequence, otherwise the results are unpredictable. This is a potential source of operator error, and should be corrected, possibly by having only one power switch.

- In some tests, we found excessive false alarms when the 100 meters near range boundary was selected. Also, these false alarms often had a velocity readout reading of $14.9 \mathrm{~m} / \mathrm{s}$, which is the maximum velocity that the radar will display.

- We experienced intermittent detection in the Coyote canyon tests where the radar tripod was sitting on a paved road and the $4.5^{\circ}$ antenna was pointing directly along the road. We speculate that multipath effects were creating zones with poor or no detection and that lifting the antenna position to a rooftop or tower would improve detection.

- The weak scan motor drive, and noise from the potentiometer are two issues already being addressed by Motorola. The system's light weight and low power consumption should be welcomed by the military. However, if it is set up on a hill top in windy weather, it must be tied down securely.

- The testing operators would have preferred to have auto-repeat on the threshold up or down function button. While this would have aided testing. for actual operation. the operator should not be adjusting the threshold more than one step at a time, starting with the default setting.

\section{Testing Conclusions}

\subsection{Useful Range}

The utility of the MSR-20 or similar radars may be limited to $2 \mathrm{~km}$ or less depending on conditions, because of the current DOE practice of using assessment cameras.

\subsection{Assessment Times}

Assessment times were evaluated using the MSR-20 for detection and an available set of pan and tilt cameras for assessment. Both the IR and CCD cameras used had a $2^{\circ} \mathrm{v}$ by $3^{\circ} \mathrm{h}$ field of view. The pan and tilt happened to be computer controlled and had a maximum available slew rate of only $6^{\circ}$ per second. Although the assessment range is limited to about $1 \mathrm{~km}$ for human targets using a $3^{\circ} \mathrm{FOV}$ thermal imager. vehicles can be easily recognized on the camera monitor at 2 to $3 \mathrm{~km}$. The wider track on the user's radar screen, and the high velocity summary data reading 
may make camera assessment unnecessary. Although Doppler assessment is much more rapid, we expect that, for most DOE users, camera assessment is more satisfying.

On a good road or dry lake bed, a vehicle that is detected at a 2420 meters range, and moving at $30 \mathrm{mph}(13.4 \mathrm{~m} / \mathrm{s}$.), will be able to reach the perimeter ( 220 meters) in 164 seconds. The MSR-20 scanning at $8^{\circ}$ per second over $90^{\circ}$, has an average revisit time of 11 seconds. It will have 4 opportunities to detect this vehicle and still provide 2 minutes of warning before the vehicle reaches the perimeter. If the vehicle is traveling at $60 \mathrm{mph}$, it will be detected at least twice with only a 1 -minute warning. These times assurne that the vehicle will be assessed only by the size of the return dot cluster and the display velocity, and not by the video cameras before sounding the alarm, since too much time will be required to bring the cameras around. These times seem reasonable, since the display velocity will be quite high and very apparent, even when in the scan mode. Of course roads that are in the alarm area should be blocked, ditched, or gated to prevent such high speeds.

If the vehicle is traveling across terrain with low vegetation such as sage brush, the speed is likely to be $15 \mathrm{mph}$ or less; providing ample opportunity to bring the camera pan and tilt around for a visual assessment. Since the maximum slew rate for our pan and tilt unit was only $6^{\circ}$ per second, the average response time for the camera movement alone was about 30 seconds and could be as much as 60 . These times assume that the camera is, on average, $180^{\circ}$ away from the target, assuming $360^{\circ}$ radar coverage and a pan and tilt that cannot continuously turn on its mount. Assuming that accurate azimuth information from the radar will eliminate the need to pan the camera in a search mode, the assessment time is mostly due to the slew rate limitation of the particular pan unit used.

\subsection{Designating Access Areas}

The MSR-20, as tested, can only accommodate access area designations outside the minimum and maximum range distances, using the range fence feature. This radar range fence feature disables the alarm beep for targets beyond the range fence setting, while all alarm spots remain displayed. This range fence can only be set at a single distance value for the full $\left(30^{\circ}\right.$ or $\left.90^{\circ}\right)$ sweep angle. The inner detection range can only be set at fixed range increments (e.g., 100 meters, 300 meters, 500 meters, 1000 meters, etc.) as configured. This means that the near coverage cannot easily conform to roads, railroad tracks, ditches, or fences. Also, in some tests we found excessive false alarms when 100 meters was selected as the near range. The source of these false alarms was not determined.

\subsection{Nearby Vehicles}

We found that this radar can be susceptible to the movement of nearby vehicles. However, when the radar was mounted on a guard tower ( 12 meters elevation) vehicles moving at a distance of 150 meters from the radar caused no alarms in a coverage sector whose near range was set to 500 meters. For installations where the radar is close to the ground, or the local traffic is farther away, a radio frequency absorbing or reflective screen could be helpful.

\subsection{Near Range Boundary}

Vehicles must not be allowed close to the selected near range boundary. This will cause false alarm beeps but with nothing showing on the operator's screen. The separation distance required 
depends on the size and shape of the vehicle, and possibly on the mounting height of the radar as well.

\subsection{Distant Targets}

We found that vehicles moving at ranges greater than the selected range mode interval did not cause alarms.

\subsection{Sector Edges}

We found that vehicles moving just outside the left or right edges of a swept sector generated alarms. The sensitivity of the radar (whether there is an alarm or not on a given target) depends on the radar cross section (ability to reflect the radar energy), the distance, and the power level at the involved portion of the beam. Since we detected walking humans at 4700 meters, there is more than enough power to detect a walking man at shorter ranges. At 1200 meters, the effective beam width was measured at $6.5^{\circ}$. This tells us that at this range, much less than $1 / 2$ power is needed to detect either people or vehicles. The effective detection beam width was even greater for a moving truck at the same range. Therefore, the area along the side of a radial sector subject to false alarms will be difficult to define. If a fence is placed along a roadway that runs radially toward the radar, and if the fence is placed far enough from the roadway to prevent false alarms from a large authorized truck, then an ATV or a person might approach along the secure side of the fence without being detected by the radar. See Figure 16.

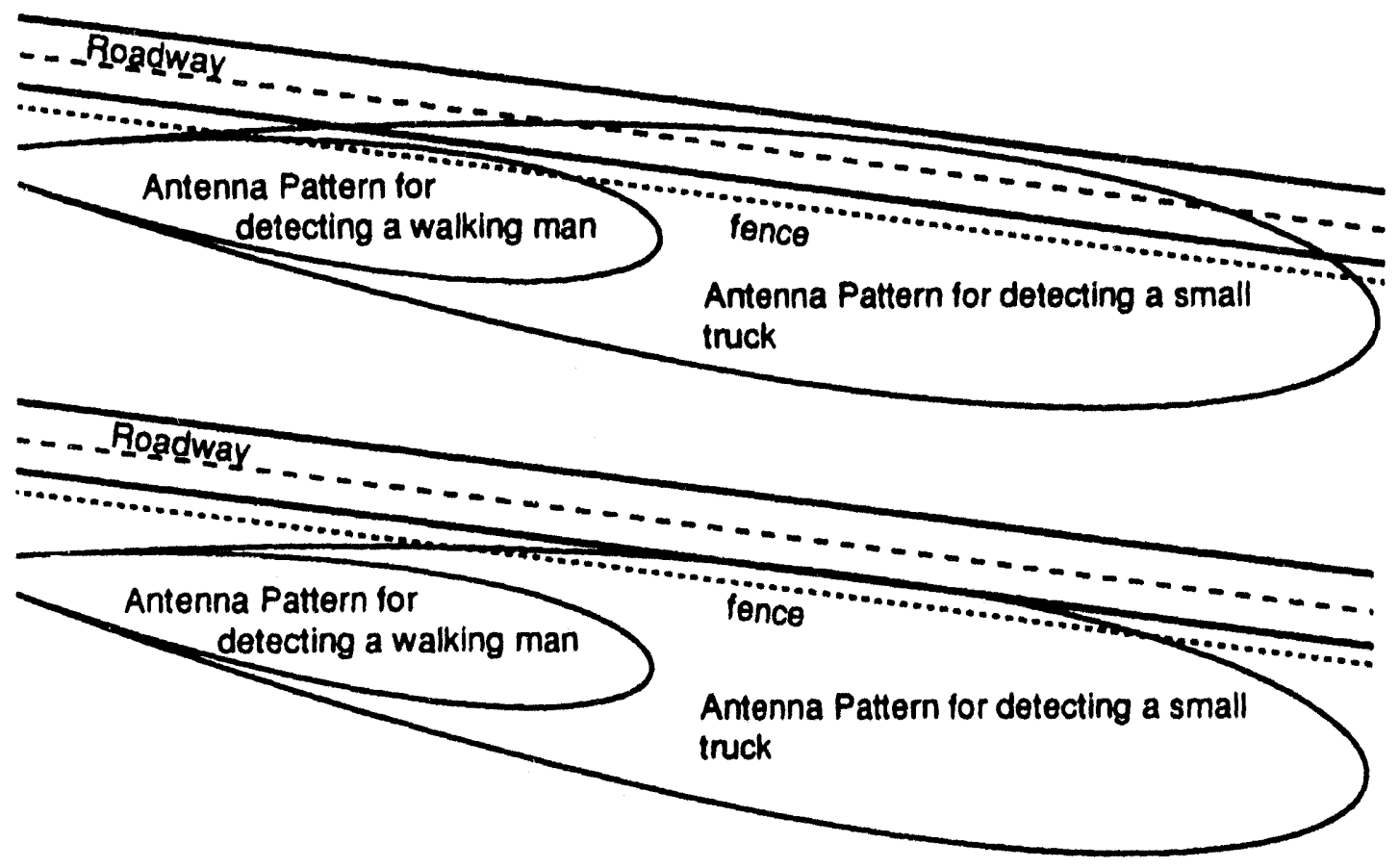

Figure 16. Radar Pattern Placement Near Access Road. 


\subsection{Antenna Beam Geometry}

\subsubsection{Available Beam Widths}

The MSR-20 is normally delivered with a $4.5^{\circ} \times 4.5^{\circ}$ beam width antenna. Most testing was done with this antenna. An $8^{\circ} \times 8^{\circ}$ antenna was also purchased to allow for comparison testing. The height of the radar beam will determine how far up the side of a hill coverage will extend. $4.5^{\circ}$ is only adequate for nearly flat conditions. If the range of interest is only a couple of kilometers, the effective vertical beam width of a $4.5^{\circ}$ antenna may be more like $6^{\circ}$, since much less beam power is needed at shorter ranges. The $8^{\circ}$ antenna may be needed for rising or upward sloping terrain, and will require less alignment precision during setup. Any area not in the radar's line-of-sight will not be covered. The $8^{\circ}$ antenna would require more power if $8 \mathrm{~km}$ detection of humans was required. However at the 2420 meters detection range there should be no extra power needed and may increase the probability of detection due to more pulse repetitions on target. Testing of the $8^{\circ}$ antenna showed good detection at 2000 meters, but did not significantly decrease the number of missed scans in 50 trials. The $8^{\circ}$ antenna beam width would allow doubling of the scan rate, which would be an advantage if $180^{\circ}$ coverage is contemplated. The new production run of MSRs is claimed to have $180^{\circ}$ capability.

\subsubsection{Pointing Assessment Cameras}

The major significance of the horizontal beam width is its impact on the assessment camera pointing accuracy. During normal operations, the sweeping beam will paint a wider or narrower set of alert dots on the screen depending upon the beam width, the range, and the reflectivity of the target. There may be an offset of this set of dots to the left or the right, depending upon the sweep direction, but this effect (due to processing time), as been mostly accounted for in the display. However, we occasionally noticed as much as $\pm 3^{\circ}$ of uncompensated "slop" that also caused left or right offsets of the displayed hits, depending upon the scan direction.

To get the most accurate angular readout in the summary information, the operator must place the cursor box over the center of the hit dot pattern. We have found that the assessment cameras, even with only a $2^{\circ}$ field of view, can be accurately placed on the target in 2 to 3 scans. Of course if the target is moving with a significant tangential motion, he may be missed. However, such fast targets usually have a very clear Doppler shift, that generates an easily assessed tone and gives a high velocity readout. This may mean that visual camera assessment is not always required.

Even so, if one wanted to improve radar azimuth pointing in order to have better azimuth data for long-focal-length cameras, and to direct the response patrol, halving the radar beam width should be considered. For a $2^{\circ}$ camera FOV, the antenna beam may have to be half its present value. This means that either the amount of processing done must be reduced, a faster CPU implemented, or the scan rate must be reduced. The scan rate could be halved if the application does not involve intruder vehicle speeds over $30 \mathrm{mph}$. Of course a narrow heam width antenna will be physically larger $\left(36^{\prime \prime}\right.$ for $\left.2^{\circ} \times 2^{\circ}\right)$ and thus will be more subject to winds. For a permanent installation, we recommend a sturdier and heavier scan drive unit that can handle wind loads without slipping or backlash. 


\subsubsection{Range Considerations}

We did most of our testing using the 1920 meter range interval, i.e., $15 \mathrm{~m}$ resolution, where the system is probably the most useful for DOE applications. The MSR can be used to cover a 3840 meter range interval to increase the warning time, but it may be hard to plan for such large areas to be devoid of legitimate traffic.

\subsection{Antenna Azimuth Positioning}

We observed that the "home" or reference position sometimes shifted. Motorola said that the zero position should never be lost; something must be broken or loose.

\subsection{Tree-line Detection}

This radar is not suitable for detecting movement inside the tree line at the edge of a woods or forest. Tests at Coyote Canyon indicated that motion of a subject behind even a sparse, dead cactus would not be detected.

\subsection{Additional General Notes and Recommendations}

- It is best to avoid having any moving targets between the radar and the covered area.

- The terrain covered should be flat or slightly howl shaped, with the land rising on the far edge.

- The radar should be mounted on high ground or on a tower for best results.

\section{Expected Battlefield Performance}

This section discusses the use of this radar for its intended military purpose and will help explain some of its characteristics. In Section 6, we discuss how it may perform in the more demanding and sophisticated DOE application.

This radar was designed by Motorola to meet military requirements for low power consumption, ruggedness, and low manufacturing cost. In doing so, it sacrifices a high visibility display, ease of use, and versatility. While it only scans a maximum of $90^{\circ}$, this is usually enough for the military who normally expect attack from only certain directions.

Personnel consulted at Sandia had little experience with such radars in military applications. However, the following information was found:

- Such radars are often used at the front lines to detect movement of the enemy from one tactical location to another, as well as to detect movement toward the friendly positions.

- The radars are often set up to cover a field of fire for guns.

- Radars of this type usually have a maximum of $90^{\circ}$ coverage and are used with other radars to provide overlapping coverage along the battle line. Obviously a ground search radar that has $360^{\circ}$ coverage has almost no application in a military situation.

- Paper maps are traditionally used with the field of fire horizontal angle and range (as well as non-line-of-sight) areas penciled in. In this manner, the radar summary information, such as range and azimuth, can be used to direct fire from the co-located guns, and by artillery in other positions. 
- Military personnel will have radar working on a wide angle coverage at a low sweep rate when enemy activity is low. When targets are detected, the angular coverage is narrowed and the sweep rate increased to get as much data as possible, including the Doppler audio and velocity signals. There is little concern about diversionary tactics being used to cause a focused response in the wrong direction.

- The motivation of military defenders is great, since their lives are threatened. When on the defense, there are a larger number of personnel available to take turns at operating the radar and listening to Doppler shift audio signatures.

- The longer reach of the military guns makes detection of personnel at 6-8 $\mathrm{km}$, and military vehicles at longer ranges entirely practical. Since friendly forces are not supposed to be in that area, assessment does not have to be as accurate. In other words, they can shoot anything that moves.

Since the MSR-20 has a display that provides all of the required information, there is no need for a computer interface for most situations. The co-located gun is the security response, and the radar provides enough assessment to distinguish military vehicles and squads of men from single animals. If a walking enemy is indicated, then the area can be swept with a machine gun.

If the radar return indicates a vehicle, a mortar or shoulder mounted rocket may be appropriate. Only recently has the possibility for casualties due to friendly fire become a major concern.

\section{Application to DOE Site Security}

\subsection{General Observations}

The MSR-20 pulse Doppler radar will detect humans and vehicles moving toward the transmitter at almost any range of interest to DOE site security managers. Motorola states that it will detect personnel un to $8 \mathrm{~km}$ away, and Sandia has confirmed detection at 4700 meters (greater distances were not attempted).

This type of radar is an excellent choice for detecting human intruders over a wide range when used by a full-time operator. It has many advantages over infrared motion detection systems and video motion detection systems. It is much less affected by rain or snow, and is not affected at all by solar heating and lighting effects. The return signature for radar is almost completely determined by the motion of the intruder's body, and not by his clothing. camoutlage. etc. Daytime detection by IR depends to a great extent on the ambient temperature and the sun angle. Any imaging system that uses motion detection against the background for intrusion detection must be arranged so that the intruder must cross the field of view. Also, Doppler radar provides good range resolution, a measurement not available from passive detection systems. 
There are nine potential drawbacks to using this radar at DOE sites:

1. Since it is an active system, the intruder may be able to use a very special detector to anticipate when the radar will be "seeing" him. According to Motorola tests, commercial $\mathrm{X}$-band police radar detectors will not respond to this radar.

2. There may be close-in interference.

3. Azimuth resolution may not be adequate for pointing assessment cameras.

4. It has a relatively long revisit time (not a problem at longer ranges).

5. It is unable to detect stationary targets.

6. Alarms cannot be disabled in irregularly shaped areas.

7. It is susceptible to nuisance alarms from animals and blowing bushes.

8. It only scans $90^{\circ}$ maximum. $\left(180^{\circ}\right.$ units may be offered in the future).

9. It has no elevation tilting adjustment.

Table 3. Comparison of DOE and DoD Needs

\begin{tabular}{|c|c|c|c|}
\hline $\begin{array}{l}\text { Detection range for } \\
\text { single human }\end{array}$ & $51) 0-2000$ meters & 500 meters to $5 \mathrm{~km}$ & 500 meters to $5 \mathrm{~km}$ \\
\hline Assessment method & $\begin{array}{l}\text { video or thermal } \\
\text { image on monitor }\end{array}$ & $\begin{array}{l}\text { hand-held thermal } \\
\text { imagers, night vision } \\
\text { goggles }\end{array}$ & $\begin{array}{l}\text { radar Doppler and } \\
\text { binoculars and/or night } \\
\text { vision goggles }\end{array}$ \\
\hline Assessment Range & $500-2000$ meters & $\begin{array}{l}\text { good assessment } \\
\text { usually needed }\end{array}$ & $\begin{array}{l}\text { solid assessment not } \\
\text { always needed }\end{array}$ \\
\hline $\begin{array}{l}\text { Control of Detection } \\
\text { Zone }\end{array}$ & $\begin{array}{l}\text { good at NTS } \\
\text { poor at Savh. Riv. }\end{array}$ & Demilitarized Zone & No man's land \\
\hline Response & $\begin{array}{l}\text { apprehend or visually } \\
\text { identify }\end{array}$ & $\begin{array}{l}\text { send roving patrol to } \\
\text { investigate }\end{array}$ & engage \\
\hline
\end{tabular}

\subsection{Assessment of Alarms}

A fundamental requirement of DOE site security is to assess the intruder as well as to detect. This prevents unnecessary security responses to nuisance alarms and helps assure that the security response is appropriate to the nature of the intrusion.

In addition to target location, the MSR-20 pulse Doppler radar presents other information to aid assessment. It contains sophisticated signal processing that provides a high degree of range resolution as well as a measure of target velocity toward or away from the transmitter. It also has an aural output of the Doppler modulation that a trained operator may be able to use to 
distinguish a walking human from an animal or a small vehicle. There may be a way to modify this radar to provide a measure of received power for each target, which would also help distinguish a vehicle from a human, and, possibly, an armed iaruder from one that is unarmed.

\subsubsection{Video Assessment}

At DOE sites, security personnel are accustomed to assessing the intruder using video: either CCD cameras with long focal length lenses, or thermal imagers. The usable assessment range for these devices is very dependent on the motivation of the operator and the dynamics of the situation.

The classic Johnson criteria states that there must be $4 \pm 0.8 \mathrm{Ips}$ (line-pairs) of resolution ( 8 pixels) across the minimum target dimension in order to recognize a military target, e.g. a human vs. a bush or fence post. This requires an appropriate HFOV and a horizontal resolution of 500 pixels or 250 line pairs (Ips). COHU, an experienced name in security cameras, has recently announced a monochrome CCD camera having a $1134 \mathrm{H}$ by $486 \mathrm{~V}$ pixel format with an average horizontal resolution of 850 TV lines. For a $16.9 \mathrm{~mm}$ focal plane image width and a $160 \mathrm{~mm}$ lens, the FOV would be 106 milliradians $(\mathrm{mr})$. This is a little less than $2^{\circ}$. The resolution is then $.124 \mathrm{mr}$. per lp. For the required resolution of $4 \mathrm{lps}$ and a target width of .5 meters, we calculate a usable assessment range of 1000 meters. This would be adequate for a security operator to distinguish visible but static targets in daylight. If the target were moving across the image, the recognition range may double.

\subsubsection{Thermal Image Assessment at Night}

At night and at ambient conditions of $70^{\circ} \mathrm{F}$ or less, the Johnson criteria for assessment may fall under the category of orientation (vertical vs. horizontal position of a man or other oblong object), where $1.4 \mathrm{lps}$ or 3 pixels in .5 meters are all that would be needed to distinguish a human vs. an animal. This is due to the additional information available from the thermal contrast with the background. With a horizontal resolution cell size of $.108 \mathrm{mr}, 3$ pixels would be $.324 \mathrm{mr}$. A .5 meter wide target would fill this angle at a range of 1500 meters. On such nights, the target would be warmer than either the vegetation or the ground, and motion clues are not as necessary. A human target which has significant motion should be recognizable out to $2 \mathrm{~km}$ or farther when using a thermal imager.

\subsubsection{Far Field Assessment of IR Images}

In far field assessment, the target occupies only 1 or 2 video lines and subtends 4 pixels or less. All of the pixels may appear to have the same intensity. This intensity may be the same as the background in general or the same as some other objects in the hackground. If the target has contrast, but no lateral movement, there is no way to distinguish it from similar sized objects in the background. Under some conditions (at night or under cold, calm, cloudy conditions) thermal contrast will be high and may allow detection to more than $3 \mathrm{~km}$. But under adverse conditions, there may be no thermal or visual contrast at all. 
Table 4. Expected Range (Meters) for Assessment

\begin{tabular}{|l|l|l|l|}
\hline & & \\
Closed Circuit TV & 2000 & $1000(2)$ & $0(3)$ \\
\hline $\begin{array}{l}\text { Low Light Level } \\
\text { TV }\end{array}$ & 2000 & $500(4)$ & $100(5)$ \\
\hline Thermal Imager & 2000 & 500 & \\
\hline
\end{tabular}

Notes: 1. high contrast against the background

2. normal civilian clothing

3. no light

4. normal site and perimeter illumination

5. starlight, or some cloud reflected light from facility

6. intruder is same temperature as background

These are rough estimates of the assessment range for the three principle assessment technologies. All situations are for a target that has some lateral movement.

\subsubsection{Image Processing Enhancements}

The human eye/brain team is more sensitive to rapid object movement than to slow motion. Shooting stars are seen even in those areas of our peripheral eyesight that have poor resolution. In Sandia's evolving safeguards technology, we have found that using frame store methods ( 2 or 3 images taken 2 or 3 seconds apart, and then played back repeatedly), will allow a human to quickly locate moving objects. This technique artificially accelerates the normally very slow motion that occurs when a distant human target is moving mostly toward the assessment cameras, and brings the speed up into the sensitive detection range of the human brain.

\subsubsection{Radar Assessment}

Assessment by radar alone is possible, but it has significant drawbacks as well as advantages. When using radar assessment, the operator must turn off the sweep and put the radar on dwell at the indicated azimuth. Vehicular targets generate a very audible tone. Walking humans can walk as slow as .6 meters per second and still be detected. The audio frequency corresponding to this speed is less than 50 cycles per second-difficult for some people to hear. It has been suggested that properly trained and motivated operators can distinguish the human gate from that of animals. If this is the case, and if occasional interruption of the radar sweep can be tolerated, then assessment far beyond $1.5 \mathrm{~km}$ may be possible. If such long range (up to $6 \mathrm{~km}$ ) detection is of interest, then more testing would be appropriate.

\subsubsection{Other Considerations}

Detecting at a range of 2000 meters means covering a sizable area of land (12 square kilometers or about 3,000 acres), where the number of animals and wind blown brush may cause a significant number of false alarms. 
Furthermore, as defined in the introduction, detection at 1 to $2 \mathrm{~km}$ will satisfy both the early warning and the stand-off weapon terrorist requirements. As a result, testing and radar evaluation has been limited to a $2 \mathrm{~km}$ range, and assessment was limited to velocity data and video imagery.

Pulse Doppler personnel radars are only suitable for cleared terrain, with grass or brush less than 2 feet in height. This is due both to the excessive false alarm rate caused by vegetation movement and the attenuation of the radar caused by such vegetation. The need to see the target for assessment also precludes much ground cover. The radar (as well as the assessment cameras) only provide line-of-sight coverage, so terrain that has extensive ditching, arroyos, or streams may not be suitable.

\subsection{Patrol Response}

In the paragraphs below, we examine the interaction between the radar system and the patrol sent to apprehend the intruder.

Assume that the target coordinates are given to a roving patrol to investigate. The radar operator can see both the intruder and the patrol until the patrol gets close enough to be heard by the intruder, who then goes for cover. A thermal imager can, especially at night, keep a watch on the intruder, even when stopped. The radar, however, will no longer detect him. Unless moved, the cursor will identify his last known location.

If patrol assessment is practical, then the useful radar detection range might be greatly increased (perhaps up to $5 \mathrm{~km}$ ). As shown in Figure 17, if the beam width is $4.5^{\circ}$ and the range is $5 \mathrm{~km}$, the patrol may have an area of 30 meters deep by 400 meters wide (from the radar perspective) to search. While the patrol is in the area searching, the intruder may be able to move undetected as a separate target by the radar. The patrol may want to carry and use their own thermal imager to help in this kind of search.

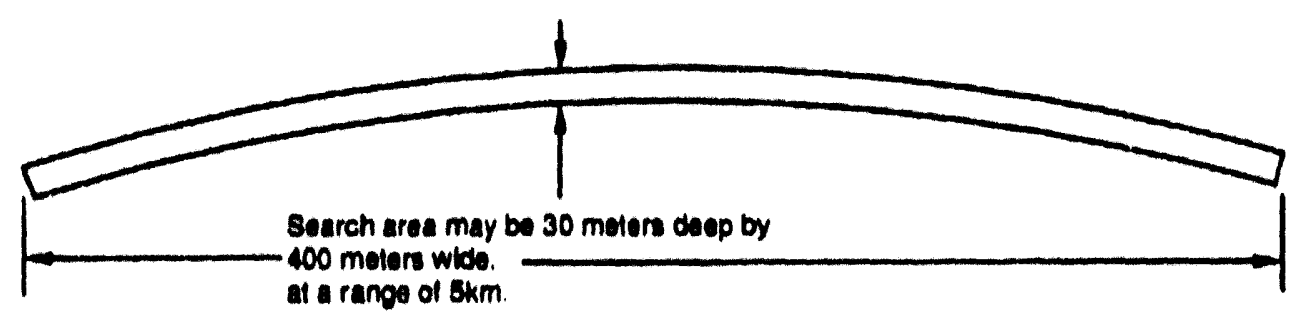

OP

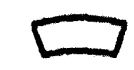

still 30 meters deep but only 40 maters wide at a ranos of 500 moton.

Figure 17. Search Areas Needed at Different Radar Ranges 


\subsection{Guldelines for Solecting Sites Sultable for Radar Use}

The following is an analysis of the characteristics of the area surrounding a security site or of a "beyond-the-fence" sector of that site. These characteristics will determine the suitability of the MSR-20 radar for use at this site or sector.

\subsubsection{Ideal Site Conditions}

Ideally, the land would be flat, barren, and hard. There would be no vegetation at all and yet there would be no sand or dust to blow up from the ground to obscure intruders and prevent assessment. There would be little or no precipitation at any time of year. There would be no roads and no traffic of any type except for periodic tests to confirm the proper operation of the radar and security center. There would be no animals in this area because there is nothing to feed on. The area would only extend out to $1.5 \mathrm{~km}$ assuming that assessment using video or thermal IR cameras is expected. Hard, dry lake beds, such as at NTS, are a typical example.

\subsubsection{Usable Site Conditions}

Usable conditions would allow low grass (6.12 inches), alive or dormant, or most any ground cover low enough not to provide temporary hiding spots during intrusion. There should be very few large rocks, trees, or ditches, since the radar must pick up target movement between these obstacles. If there are too many such obstacles, assessment may not be reliable. and the alarms may be discounted as being caused by animals. There may he gophers, rabbits and eoyotes. The thresholds could be adjusted to try to discriminate against the animals in favc, of intruders but this is not usually recommended since this could result in unacceptably low detection sensitivity.

The number of coyotes or other large predators or dogs may have to be controlled.

Discrimination between humans and animals based on the radar return (or threshold) may not be reliable, especially over a range variation such as 500 to 2500 meters.

\subsubsection{Marginal Site Conditions}

Conditions may be considered marginal when the false alarm rate (due to blowing vegetation or animals) becomes high enough that some operators will start to discount all alarms as false alarms, or where the detection probability is lowered significantly due to the presence of trees. rock, buildings, berms, ditches or other terrain features.

\subsubsection{Unsuitable Site Conditions}

If there is a public road near the site. any area beyond the road is not a candidate for early warning. It would be easier for a vehicle to have a "flat tire" or "run out of gas" or be abandoned on the public road by the intruder than for him to cross the field beyond. If the road is not a public road, but in constant use by on-site personnel, side lobe returns may cause false alarms. Mounting the radar high enough may prevent such alarms if the roadway is only a few tens of meters away. There may also be a way of shielding these signals with a radar "screen".

If the surrounding area is covered with trees or brush, neither the radar nor the assessment cameras would be useful. If the area is open, hut full of cows, cieer, or other animals, an alert human operator (guard) will be needed constantly. Detection plays no role in this situation, only constant assessment, and automatic assessment has not progressed far enough to take on this task. The radar will be of no use in this situation. 
If the area topography contains hidden approach paths, the radar alone will not be enough. In this case, point and line sensors, or local bi-static motion detection systems, may have to be installed. The big disadvantage of these systems is that they are both active and unprotected, easily located and destroyed. Such systems work best for short term protection in a temporary location.

\subsection{Weather Effects}

Besides the wind effects already noted, precipitation may have some effect-another reason why this radar would be more effective in the drier western areas. We did not test this radar in rain, snow, or fog. Theory indicates that any radar at this frequency will be little affected by moderate rain or snow, especially at these short ranges, and not at all by fog or low clouds. Wind driven rain may cause enough Doppler shift to cause alarms but this was not tested.

Motorola states that "Operation in inclement weather including rain is expected to be a routine implementation of the MSR. The equipment is designed to operate under conditions of not greater than 4 millimeters of rain per hour without system degradation. In a non-operating configuration, the radar will withstand up to $10 \mathrm{~mm}$ of rain per hour."2

\subsection{Radar Modifications for DOE Applications}

\subsubsection{Proposed Operation with Unmodified Radar}

It would be difficult to use this radar to provide early warning at most DOE sites. It would be most effective for sites that have and use guard towers. These towers are located along the sides or at the corners of the double fence PIDAS (Perimeter Intrusion, Detection, and Assessment System). The radar could be installed on these towers to provide early warning to the security operator. The dish could be mounted on the roof or the railing of the tower cab. Three such radars and displays could be used for every tower that is mounted at a perimeter corner, and two for each tower mounted in the center of a perimeter side. The LCD radar control panel could be mounted on or in the guard console, and he could manually transfer the information summary data (azimuth and range) to his copy of the site map in ordet to provide intercept coordinates to the response force. As an alternative, he could radio in the radar ID number along with the azimuth and range numbers to the Central Security Command room. The CSC would translate the information and dispatch the response.

Binoculars are usually included as standard guard tower equipment. If they were to be used to assess alarms generated by a radar, some means of pointing them would be needed. The best way would be to have an LED azimuth display built into the optics. If such a modified pair of hinoculars, high-powered telescope, or night vision 'scope were mounted on a stanchion and pivot, then a shaft encoder could be used to generate the data for this display. Additional radar data processing would help in accurate aiming especially for long range assessment.

By locating these radars on guard towers, the situation more nearly duplicates the military environment that these radars were designed for. The setting is labor intensive, and somewhat vulnerable, but very flexible. The radars would require no modification. Their elevation and location close to the perimeter precludes interference from patrol vehicles or other activity inside

2 A Detailed Description of ihe Modular Surveillance Radar MSR-20

Motorola, Inc., Tactical Electronics Division, 1991 
the site perimeter. The elevation of the radar antenna on the roof of the tower cab minimizes the non line-of-sight (LOS) areas, and would be expected to greatly reduces multipath radar effects (areas of no detection, multiple targets, etc.)

The disadvantage of this type of installation is that the number of guards, towers, and radars is large, usually a minimum of 4 towers and 12 radars for a square site that would be approximately $2 \mathrm{~km}$ on a side. Note that if the radar had $140^{\circ}$ coverage, only 8 radars would be needed. Motorola is now working on a model that has $180^{\circ}$ coverage.

Another advantage of guard tower installation is that the radar range is a fair match for the assessment range of regular and night vision binoculars. These direct view devices are cheaper. more reliable, and have much better resolution and useful range than their video counterparts. However, thermal imagers will still have to be used at many locations for night assessment, where lighting from nearby cities or the facilities is insufficient.

Providing early warning detection despite access roads is a problem not encountered in most military applications. The three approaches considered here are:

1. arranging or rearranging the access road, towers, and radars so that the roadway, shoulders. and right of way are not illuminated by the radar beam

2. ignoring the alarms from the legitimate traffic, or

3. setting up an access control point some distance $(2 \mathrm{~km})$ fron the normal site perimeter.

All of these potential solutions have serious drawbacks.

In the first solution, arranging the coverage angle for the radar is a function not only of the radar scan and beam width, but the range, size, and reflectivity of the target. At the ranges of interest $(<2 \mathrm{~km})$ vehicles on the approach road will be detected as if the beam were 50 to 100\% wider than normal. If the radar scan coverage is stopped far enough away from the access road to prevent false alarms from traffic, intruders may be able to approach the perimeter areas near the gate. At night they will be detected neither by the radar nor by site personnel traveling the access road. Gate personnel may see them only if they are constantly reminded to watch.

In the second solution (ignoring alarms), if the towers and radars are set up to cover the access road, then security personnel will just have to tolerate and ignore the alarms that occur in that area. A corridor could be marked on an overlay on the control panel I.CD alarm map display. The big drawback with this method is that the guard must force himself to examine the display for each alarm beep that he hears, and then somehow identify the authorized traffic through some kind of coded messages. so that unauthorized intrusions can be identified. This method is probably impractical.

The third solution does not offer much over the second, except that the legitimate traffic has already heen identified. The authorized traftic could be grouped in convoys. This would be a major inconvenience for everyone.

\subsubsection{Proposed Operation with Modified Radar.}

The radar could be modified so that the azimuth and range (memory location) of the cursor box would be passed (RS-232) to a computer that had an elevation look-up table, an azimuth offset computation, and a controller for the assessment camera pan and tilt. The operator would still 
have to manually adjust the cursor, in order for the system to know which alarm to assess. The central computer could handle 5 or 6 radars feeding informution into a single pan and ill, of if could feed 5 or 6 pan and tilt mounts co-located with the radars. The CPU would switch the appropriate cameras to the monitors and frame storage/replay units, and feed alarm information to the site security situation display map. This method is also foo labor intensive and is not recommended.

\subsubsection{Fully Integrated Radar System}

In this approach, the hardware would be arrayed as above, hut there would he no individual radat operators. Instead. a centralized computer with a target clustering and tracking algorithm would be used to process the alerts from all of the radars. This would reduce halse alarms, antomatically place likely targets on the central security screen, and direct the assessment pan and ill camefas. This security screen would also display a map of the site and surrounding area 6 holp direct the response team. This would be our recommendation if the other aspects of the sile (such as terrain and access) qualify for the use of this radar. An automated detection system will not be as effective as an alert, trained radar operator, but will be much helter than one who is inattentive or bored. In this method. the computer would gather the velocity information fron each hit and use it in the tracking and clustering algorithm. See Figure 18.

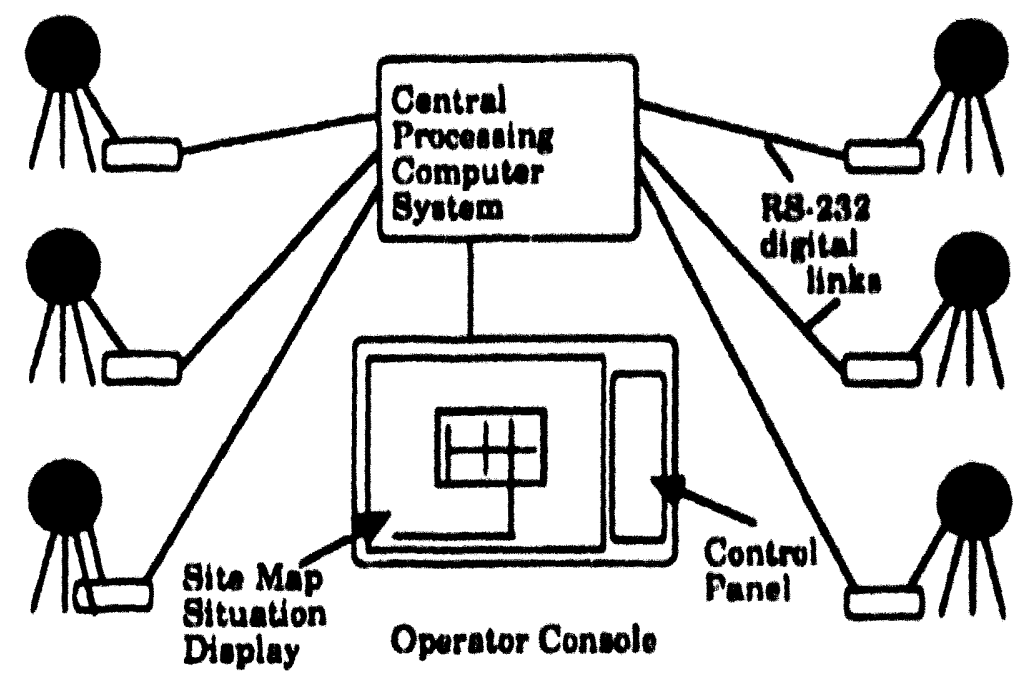

Figure 18. Block Dlagram of a Centrallzed Alarm Proceanor and True Map Dlaplay

Besides the elimination of manual radar operation, there are lwo other advantayes fo centralized alurm processing.

1. Alarm coordinates can be automatically converted from the radar asimuth and fange data to normal rectangular map coordinates or sector numbers, and

2. Alarms along legitimate approach routes or access rouds can be masked out hetore alertiny the operator and showing alarms on the central sile situation security map display. 
This adaptation involves modifying the software for the radar to provide all of the alarm data stored for each sweep to an external computer through an RS-232 or parallel interface link. When used in this manner, the assessment function now provided on the radar LCD screen by moving the box cursor with the arrow keys would be replaced by a cursor on the site map graphics moved by a mouse.

More sophisticated software functions can be added as well. One possibility is to use tracking algorithms to further reduce the false alarms and the amount of data the operator must deal with. Targets that had about the same radar return, velocity, and position over several scans would generate a much higher priority alarm than other data combinations. However, tracking algorithms must be used cautiously. Some "good" tracking software may actually reduce performance if the intruder does not follow the type of movements anticipated in the algorithm. Lost tracks may also be a problem because of the low update rate.

The problem of providing proper coverage near access roads remains. Vehicles will generate alarms along a path that is much wider than the radar beam width. If all of these alarms are masked out, then the system may not be sensitive to walking intruders that are near but not on the road.

On the other hand, if additional alarm processing can be used, then the centroid of the alarm grouping can be taken as the actual intruder position. This position may be further refined to determine if the target is most probably located on an access road.

This refinement of the target aziniuth will also greatly help in the accurate and automatic aiming of the long focal length cameras that must be used for early warning assessment. These cameras should have a field of view that is as narrow as $1^{\circ}$. Even then, because the intruder may be coming toward the camera, rather than across the camera field as in a PIDAS, visual detection may be very difficult. Some form of sequential image store and rapid playback may be needed to notice and assess distant intruders on the monitor.

\subsection{Site Coverage}

By increasing the scan angle of this radar from $90^{\circ}$ to $180^{\circ}$ or $270^{\circ}$, only one radar would be needed at each guard tower. Also, two such radars, each with $180^{\circ}$ scan could be placed at the center of a small site $(<2 \mathrm{~km}$ on a side) and yet give good early warning coverage. The problem with such an installation is that false alarms might be generated by traffic inside the perimeter fence. Such cars and trucks will cause moving radar reflections that generate "legitimate" alarms appearing to come from outside the perimeter.

To cover normal approach paths. overlapping $90^{\circ}$ coverage may be satisfactory. If you try to cover $180^{\circ}$ with little overlap. some targets may be walking tangentially to the radar, and not have enough radial velocity to be detected. See Figure 19B.

To cover a site that is only 1 or $2 \mathrm{~km}$ across, $360^{\circ}$ coverage from a single radar, as shown in Figure 19A. might be the least expensive solution, but has four times the revisit time 45 seconds average.) This time could be almost halved by using the smaller antenna with the $8^{\circ}$ beam width.

$A$ hig disadvantage of placing the radar in the center of the area may be false alarms caused by the on-site vehicular traffic. A very tall tower would he' this is illustrated in Figure 20. 


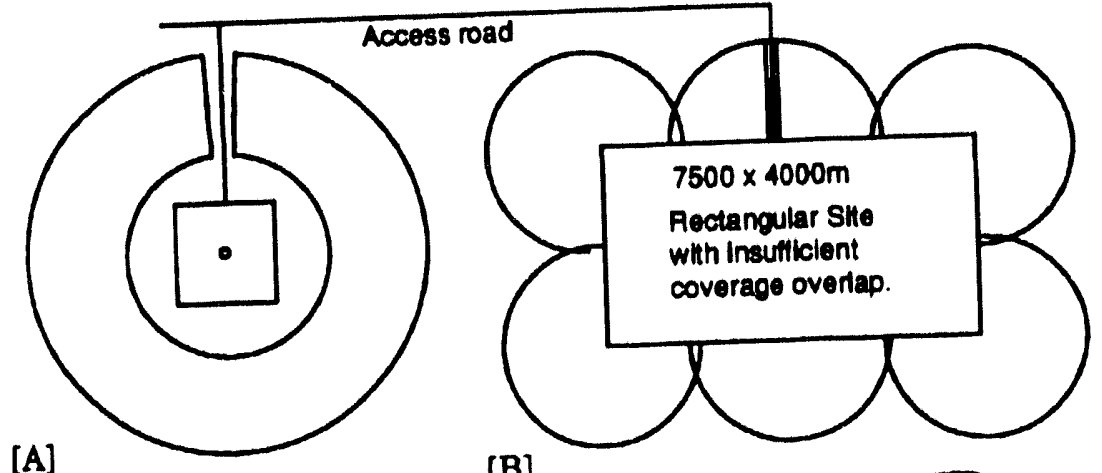

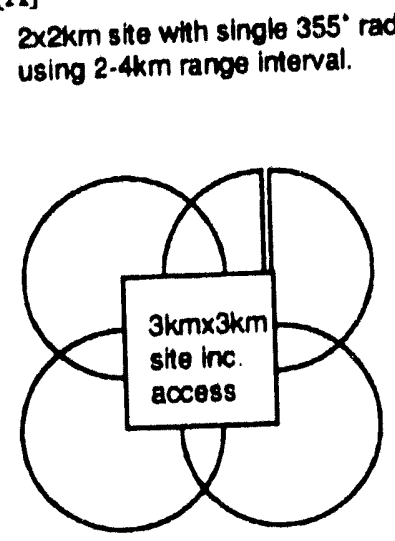

[C]
[B]

[D]

Figure 19. Radar Location and Coverage for Typical Sites

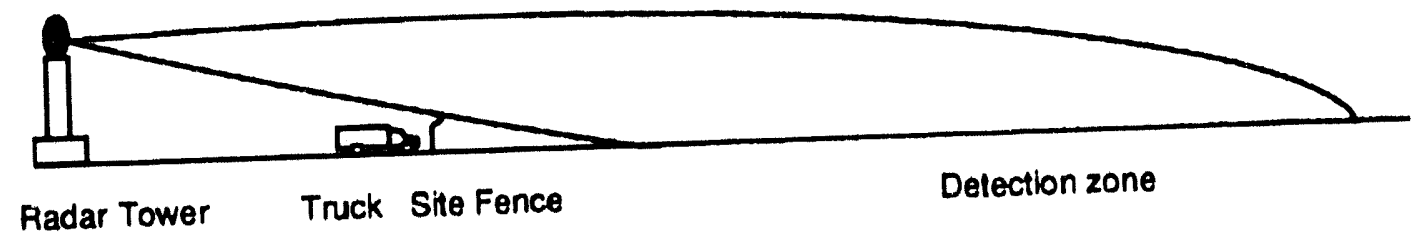

Figure 20. Single Radar On Tall Tower (Drawing not to scale)

If one attempts to place the radar and assessment cameras on a central tower at a small area (less than $2 \mathrm{~km}$ on a side), there may be a problem with on-site vehicle traffic near the perimeter. Such traffic will reflect radar returns from stationary targets that will appear to be coming from outside the perimeter. It may be possible to solve this problem by placing a low screen around the radar antenna as shown in Figure 21 to reduce this problem. For example, if the radar were placed in the center of the roof of a large building, then a metal lath or hardware cloth shield could possibly be erected around the edge of the roof to prevent close-in Doppler returns. A similar solution has been observed in screening satellite dishes, but we have no experience in applying this solution to radar antennas. 
If the assessment IR cameras and video cameras are located near the radar, then assessment will be more difficult than if these cameras were located at the perimeter. This difficulty stems from the increased target range. At night the possibility exists of on-site and perimeter lighting "blinding" the low light level cameras (if used).

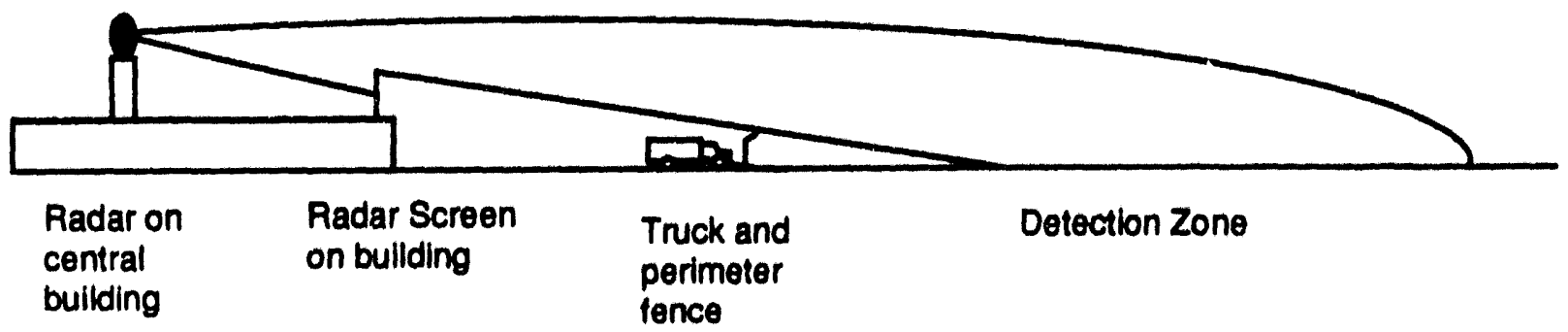

Fig. 21. Radar Screen to Reduce False Alarms from Perimeter Traffic

Early warning at DOE sites, on the other hand, may require detection over almost $360^{\circ}$, except for access road(s). The detection will be from the fenced area outward, and there may be significant vehicular and pedestrian traffic inside this fence. The radar must either be set up to exclude the access roads (usually not possible or practical), or the alarms generated by the radar covering these roads must be gated out using software and computer overlay maps.

If the site is small, so that the radar and assessment camera suite can be centrally located, then up to $1 \mathrm{~km}$ of range may be lost before getting to the surrounding detection zone. Because of this problem, and the problem of extraneous radar returns and reflections from on-site traffic, a centrally located sensor tower is probably not a good idea. The radars and assessment cameras should be located at the perimeter, and probably at the corners of the perimeter for the best coverage. See Figure 19C and 19D above for two possible configurations.

To cover a square site that measures $3 \mathrm{~km}$ on a side (for example), at least five perimeter-located radars should be used as shown in Figure $19 \mathrm{C}$. One radar would have a $90^{\circ}$ coverage, one $180^{\circ}$, and the other three radars would need $270^{\circ}$ coverage. These individual radars can be better adjusted to compensate for uneven terrain. The main advantage here is that each radar is located beyond the on-site perimeter traffic.

\section{Application to Specific (Typical) DOE Sites}

\subsection{NTS Vertical Hole Tests}

These tests are conducted in low-lying flat areas, with either a dry lake bed or dead grass and sagebrush covered desert flats. Neither the required detection range for intruder safety, nor the response time of intercept patrols are known. It is not known whether a 1500 meter assessment range is adequate. Vehicle velocities on the dry lake bed are probably too high to allow for patrol intercept. For bare, grassy or sagebrush covered flats, helicopter intercept could be fast enough. 


\subsection{NTS Tunnel Shots}

For Black Mesa tests, we believe that the terrain is too wooded and undulating to provide coverage with radar alone. The ditches, arroyos and ravines would have to be monitored by placing unattended, $\mathrm{rf}$-coupled, gruund sensors at strategic locations.

\subsection{Pantex}

This site is typical of a location where there is at least one public access road close to the site boundary. Pantex has a storage area and a working area that are surrounded by high security, perimeter intrusion detection and assessment system (PIDAS) double fences. The PIDAS sectors are also covered by guard towers. These could be used to mount radars in order to extend early warning. The big problem here is that much of the site surrounding these high security areas has a lot of legitimate traffic. There are isolated areas that could be covered by modified radars, but there is little incentive for an intruder to use these areas in his approach. A terrorist could easily be disguised as a contractor employee, appearing to do maintenance, etc.

While the radars could be set up to look outside the site boundary only, there are a number of roads that are unrestricted to the public. If the radars are deployed beyond these roads, then they would have no protection against vandalism, and could be bypassed by an intruder using the close in roads.

The use of radar to provide early warning at Pantex does not seem feasible.

\subsection{Hanford}

The Hanford Reservation has a large number of individually secured technical sites. Although the public has access to this land, it is government property and buffer zones can be more easily established around many of these sites. The land is often fairly flat and usually covered with low sage brush. This sage brush stands 2-3 ft high, and would not block radar detection of a walking human at ranges under $2 \mathrm{~km}$. However, if the intruder suspected that a radar was being used, he could move intermittently and hide behind these low bushes. Furthermore, the Hanford reservation has gullies or arroyos that may provide hidden approach paths.

Deer and other animals range this land however, and there is also a significant rodent, rabbit, and coyote population. Dr. V.W. Howard of NMSU states that it is normal to have .5 to 1 coyote per square mile in similar unpopulated areas near Albuquerque. The rabbit population can be much higher, being 20-30 animals in a 200 meter wide swath a mile long. Thus it seems probable that there could be a high nuisance alarm rate due to the animal population alone.

The MRS-20 radar may find application in this area for early warning and to detect terrorists with stand-off weapons. However, 4 or 5 radars would have to be deployed at each site to provide coverage on all sides plus the area near the normal access. Fences with motion detection switches (MDSS) may have to be installed on either side of the access road(s). If the access road does not lead straight toward the radars that cover that area, either the road must be moved, the radars relocated, or the radars modified. If there are other roads within $2 \mathrm{~km}$ of the site, they would have to be rerouted, or blocked off, or else the security plan (response time) would have to allow for less early warning. 


\subsection{Idaho INEL}

This site is reported to have terrain and vegetation nearly identical to that at Hanford. It is known to have a significant deer population, which may cause an intolerable false alarm rate. Therefore, the advantages and problems of the application of this radar to Hanford should apply here as well.

\subsection{Rocky Flats}

From a cursory examination of topographic maps of this site, this site may be too rough for good radar coverage.

\subsection{Eastern and Southern DOE Sites}

These sites (such as Savannah River and Oak Ridge) are often covered with woods except for a small area around the reactor, processing, or storage sites. There are access and maintenance roads running everywhere. Hunters and fishermen are allowed on the reservation, and public highways pass through the reservation. The edge of the woods may be far enough to provide a significant early warning, but it is doubtful that an unauthorized intruder would approach from that direction. He is more likely to enter the road system elsewhere, and then drive right up to the site fence. The use of radar at these sites is not recommended unless larger clearings can be cut and maintained.

\section{Ability to Detect Terrorists with Stand-off Weapons}

The MSR-20, or any other Doppler radar, will detect an intruder only as long as he is moving and not hidden behind brush, etc. In those areas where the intruder must advance toward the facility within line-of-sight, he will certainly be detected If he can approach up the back side of a hill, through tall crops, or through the woods, detection is unlikely. He would not be expected to move rapidly or far enough when partially exposed at the tree line or the top of the hill to allow reasonable detection.

\section{Assessment lssue Summary}

Accurate assessment is a cornerstone of DOE security. We cannot shoot first and ask questions later. Accurate assessment also allows for an appropriate response and helps prevent government embarrassment, or the injury or death of accidental intruders.

- Using the MSR alone, detection of single intruders is possible out to and beyond 4700 meters; but assessment at this distance will take considerable experience and training of the radar operator. The radar may not be able to tell how many attackers are in the group, or if they are armed. The operator may be able to distinguish walking men from vehicles.

- If video imaging is used (CCD, LLTV, or thermal IR), much better assessment is possible, but only at much shorter ranges (at most $2 \mathrm{~km}$, in our opinion). To be able to assess at longer ranges, very long focal length optics are needed along with a sturdy, accurate and smooth pan and tilt mechanism. To reduce the search time for the narrow $\left(1^{\circ}\right)$ FOV required, the target azimuth must be known with more accuracy than is currently available with the MSR-20 radar. 


\section{Conclusions}

The Motorola MSR-20 is a sensitive intrusion detection radar. It contains sophisticated Doppler return processing and a reasonably simple operator control panel. We were impressed by the reliable detection of walking intruders at all the ranges tested, and by the very low FAR that could be attributed to the radar itself.

Although we found that it would detect even single personnel at significant ranges as advertised. it is sensitive to false alarms in areas having blowing vegetation. We would logically expect animal populations to also cause nuisance alarm problems. It is designed for operation by dedicated and trained personnel, and must be modified before it could be integrated into any existing or proposed site security system.

This radar would seem to have its best application to isolated sites in the western US where there is little ground cover. Since it can cover thousands of acres, there may be a high nuisance alarm rate from animals. Also, the false alarms from blowing vegetation may force the operator to raise the detection threshold to the point where intrusion detection is no longer possible.

AII DOE sites have access routes that must somehow be accommodated by the radar. This is usually not the case for military applications, where enemy approach (and radar coverage) is only from one direction.

Computer processing of the alarm data may be used to reduce the security operator workload. If' large targets cause many returns, these should be clustered into a single alarm. Memory-mapped exclusions zones should be used to prevent nuisance alarms from areas having authorized traffic.

It also became evident that there will be few civilian applications that can take advantage of an $8 \mathrm{~km}$ detection range.

Because of these factors, successful implementation of the MSR-20 requires that the site he carefully selected, and terrain and/or ground cover modification of the site may have to be considered. To apply the MSR-20 successfully, full individual site analysis, with complete system planning and engineering may he required. 


\section{DISTRIBUTION:}

U.S. Department of Energy

Attn: O. Dan Smith

Office of Safeguards and Security

P.O. Box A / SA-134

Germantown, MD 20874

U.S. Department of Energy

Attn: Carl Pocratsky

Office of Safeguards and Security

P.O. Box A / SA-134

Germantown, MD 20874

U.S. Department of Energy

Attn: Darryl Toms

Office of Safeguards and Security

P.O. Box A / SA-121

Germantown, MD 20874

9500 D. S. Miyoshi

9503 J. W. Kane

9504 R. W. Moya (5)

9508 R. F. Davis

9521 D. E. McGovem

9531 D. J. Gangel

9533 D. C. Hanson

9538 S. Ortiz

9538 C. L. Beer

9538 S. A. Nichols

9538 R. A. Ortiz

9538 J. D. Patrick

9538 D. A. Pritchard

9549 B. J. Steele

9549 R. G. Vigil

9549 L. D. Miller

9549 C. E. Ringler

9549 T. P. Malone

9549 D. D. Spencer

9549 Sensors Library (5)

9561 J. D. Williams

9561 H. D. Arlowe (15)

9561 J. B. Pletta

9616 J. P. Rebeil
8523-2 Central Technical Files

7141 Technical Library (5)

7151 Technical Publications

7613-2 Document Processing for DOE/OSTI (10) 

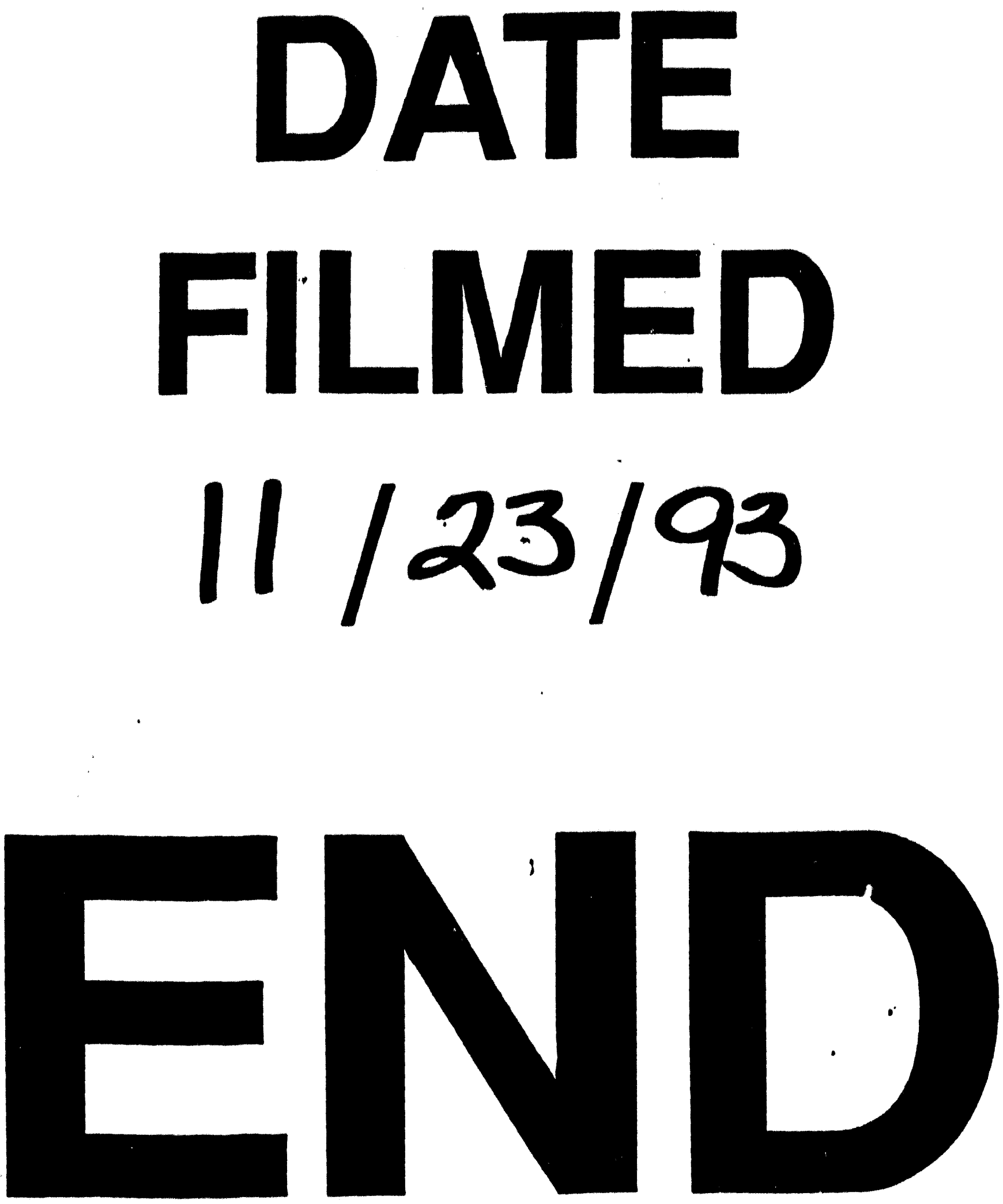
